\title{
Conventional Chemotherapeutic Drug Nanoparticles for Cancer Treatment
}

\author{
Loredana Serpe
}

1.1

Introduction

Chemotherapy is a major therapeutic approach for the treatment of localized and metastasized cancers. The selective increase in tumor tissue uptake of anticancer agents would be of great interest in cancer chemotherapy since anticancer drugs are not specific to cancer cells. Routes of administration, biodistribution and elimination of available chemotherapeutic agents can be modified by drug delivery systems to optimize drug therapy.

This chapter focuses on progress in targeted treatment of cancer through the delivery of conventional anticancer agents via microparticulate drug carriers as nanoparticles. Briefly, nanoparticles may be defined as submicronic colloidal systems that are generally made of polymers and, according to the preparation process used, nanospheres (matrix systems) or nanocapsules (reservoir systems) can be obtained. The drug can either be directly incorporated during polymerization or by adsorption onto preformed nanoparticles [1].

The first part of this chapter pays particular attention to cancer as a drug delivery target and to the development of different types of nanoparticles as drug delivery device. The interaction of drug carrier systems with the biological environment is an important basis for designing strategies; these systems should be independent in the environment and selective at the pharmacological site. If designed appropriately, nanoparticles may act as a drug vehicle able to target tumor tissues or cells, protecting the drug from inactivation during its transport. The formulation of nanoparticles and physicochemical parameters such as $\mathrm{pH}$, monomer concentration, added stabilizer and ionic strength as well as surface charge, particle size and molecular weight are important for drug delivery.

For instance, poly(acrylamide) nanocapsules, due to their polymeric nature, are stable in biological fluids and during storage, and can entrap various agents in a stable and reproducible way but, since they are not lysed by lysosomal enzymes, their clinical application is restricted [2]. The development of biodegradable polymers by the polymerization of various alkyl cyanoacrylate monomers and the association of anticancer agents with these poly(alkyl cyanoacrylate) polymers has

Nanotechnologies for the Life Sciences Vol. 6

Nanomaterials for Cancer Therapy. Edited by Challa S. S. R. Kumar

Copyright (C) 2006 WILEY-VCH Verlag GmbH \& Co. KGaA, Weinheim

ISBN: 3-527-31386-9 
2 1 Conventional Chemotherapeutic Drug Nanoparticles for Cancer Treatment

afforded further improvement. These colloidal drug carriers are biodegradable and can associate with various drugs in a non-specific manner. The binding capacity of these nanoparticles to dactinomycin (90\%), vinblastin (36-85\%) and methotrexate (15-40\%) exceeds that of these drugs incorporated in liposomes [1].

Furthermore, certain types of nanoparticles are able to reverse multidrug resistance - a major problem in chemotherapy - and selectivity in drug targeting can be achieved by the attachment of certain forms of homing devices, such as a monoclonal antibody or lecithin [3].

Finally, the last part of the chapter reports some of the most significant and recently developed nanoparticle formulations for the delivery of specific anticancer agents.

\section{2}

\section{Cancer as Drug Delivery Target}

Tumor blood vessels have several abnormalities compared with physiological vessels, such as a relatively high proportion of proliferating endothelial cells, an increased tortuosity and an aberrant basement membrane formation. The rapidly expanding tumor vasculature often has a discontinuous endothelium, with gaps between the cells that may be several hundred nanometers large [4, 5].

Macromolecular transport pathways across tumor vessels occur via open gaps (interendothelial junctions and transendothelial channels), vesicular vacuolar organelles and fenestrations. However, it remains controversial which pathways are predominantly responsible for tumor hyperpermeability and macromolecular transvascular transport [6].

Tumor interstitium is also characterized by a high interstitial pressure, leading to an outward convective interstitial fluid flow, as well as the absence of an anatomically well-defined functioning lymphatic network. Hence, the transport of an anticancer drug in the interstitium will be governed by the physiological (i.e., pressure) and physicochemical (i.e., composition, structure) properties of the interstitium and by the physicochemical properties of the molecule itself (i.e., size, configuration, charge, hydrophobicity). Physiological barriers at the tumor level (i.e., poorly vascularized tumor regions, acidic environment, high interstitial pressure and low microvascular pressure) as well as at the cellular level (i.e., altered activity of specific enzyme systems, altered apoptosis regulation and transport based mechanisms) and in the body (i.e., distribution, biotransformation and clearance of anticancer agent) must be overcome to deliver anticancer agents to tumor cells in vivo [1].

Colloidal nanoparticles incorporating anticancer agents can overcome such resistances to drug action, increasing the selectivity of drugs towards cancer cells and reducing their toxicity towards normal cells.

The accumulation mechanism of intravenously injected nanoparticles in cancer tissues relies on a passive diffusion or convection across the hyperpermeable tumor vasculature. Additional retention of the colloidal particles in the tumor inter- 
stitium is due to the compromised clearance via lymphatics. This so-called "enhanced permeability and retention effect" results in an important intratumoral drug accumulation that is even higher than that observed in plasma and other tissues [7]. Controlled release of the drug content inside the tumoral interstitium may be achieved by controlling the nanoparticulate structure, the polymer used and the way by which the drug is associated with the carrier (adsorption or encapsulation).

However, anticancer drugs, even if they are located in the tumoral interstitium, can have limited efficacy against numerous tumor types because cancer cells are able to develop mechanisms of resistance. Simultaneous cellular resistance to multiple lipophilic drugs is one of the most important problems in chemotherapy. This drug resistance may appear clinically either as a lack of tumor size reduction or as the occurrence of clinical relapse after an initial positive response to antitumor treatment. Multidrug resistance is mainly due to overexpression of the plasma membrane P-glycoprotein, which is capable of extruding various generally positively charged xenobiotics, including some anticancer drugs, out of the cell. Multidrug resistance is always multifactorial when other mechanisms can be associated with this drug efflux pump in cancer cells, such as enzymatic function modification (topoisomerase, glutathione S-transferase) or altered intracellular drug distribution due to increased drug sequestration into cytoplasmic acidic vesicles [8]. P-glycoprotein probably recognizes the drug to be effluxed out of the cancer cell only when the drug is present in the plasma membrane, and not when it is located in the cytoplasm of lysosomes, after endocytosis. Many cancer cell types can develop resistance to doxorubicin, which is a P-glycoprotein substrate, therefore incorporation of this compound into nanoparticles to reverse multidrug resistance P-glycoprotein mediated has been extensively investigated [9]. Certain types of nanoparticles are able to overcome multidrug resistance mediated by the $\mathrm{P}$ glycoprotein, such as poly(alkyl cyanoacrylate) nanoparticles [10].

The delivery of anticancer agents to a highly perfused tumoral lesion and the tumor cells response have been described through the development of a twodimensional tumor simulator with the capability of showing tumoral lesion progression through the stages of diffusion-limited dormancy, neo-vascularization and subsequent rapid growth and tissue invasion. Two-dimensional simulations based on a self-consistent parameter estimation demonstrated fundamental convective and diffusive transport limitations in delivering anticancer drug into tumors via intravenous free drug administration or via $100 \mathrm{~nm}$ nanoparticles injected into the bloodstream, able to extravasate and release the drug into the tumoral tissue, or via 1-10 $\mathrm{nm}$ nanoparticles, able to diffuse directly and target the individual tumor cell. Even with constant drug release from the nanoparticles, homogenous drug-sensitive tumor cell type, targeted nanoparticle delivery and model parameters calibrated to ensure sufficient drug or nanoparticle blood concentration to kill all cells in vitro, analysis shows that fundamental transport limitations are severe and that drug levels inside the tumor are far less than in vitro. This leaves large parts of the tumor with inadequate drug concentration. Comparison of cell death rates predicted by simulations reveals that the in vivo rate of tumor reduction is 
several orders of magnitude less than in vitro for equal chemotherapeutic carrier concentrations in the blood. Small nanoparticles equipped with active transport mechanisms would overcome the predicted limitations and result in improved tumor response [11].

1.3

Nanoparticles as Anticancer Drug Delivery System

The fate of a drug after administration in vivo is determined by a combination of several processes, such as distribution, metabolism and elimination when given intravenously or absorption, distribution, metabolism and elimination when an extravascular route is used. The result depends mainly on the physicochemical properties of the drug and therefore on its chemical structure. In recent decades, much work has been directed towards developing delivery systems to control the fate of drugs by modifying these processes, in particular the drug distribution within the organism. Nanoparticles loaded with anticancer agents can successfully increase drug concentration in cancer tissues and also act at cellular levels, enhancing antitumor efficacy. They can be endocytosed/phagocytosed by cells, with resulting cell internalization of the encapsulated drug. Nanoparticles may consist of either a polymeric matrix (nanospheres) or of a reservoir system in which an oily or aqueous core is surrounded by a thin polymeric wall (nanocapsules). Suitable polymers for nanoparticles include poly(alkyl cyanoacrylates), poly(methylidene malonate) and polyesters such as poly(lactic acid), poly(glycolic acid), poly( $\varepsilon$-caprolactone) and their copolymers [3].

Nanoparticles of biodegradable polymers can provide controlled and targeted delivery of the drug with better efficacy and fewer side-effects. Lipophilic drugs, which have some solubility either in the polymer matrix or in the oily core of nanocapsules, are more readily incorporated than hydrophilic compounds, although the latter may be adsorbed onto the particle surface. Nanospheres can also be formed from natural macromolecules such as proteins and polysaccharides, from non polar lipids, and from inorganic materials such as metal oxides and silica [3].

As cancer chemotherapeutic agents are often administered systemically, numerous biological factors, associated with the tumor, influence the delivery of the drugs to the tumors. Consequently, drug delivery systems to solid tumors have been redesigned and, subsequently, injectable delivery systems (i.e., solid lipid nanoparticles) have been developed as an alternative to polymeric nanoparticles for adequate drug delivery to solid tumors [12].

The introduction of synthetic material into the body always affects different body systems, including the defense system. Synthetic polymers are usually thymusindependent antigens with only a limited ability to elicit antibody formation or to induce a cellular immune response against them. However, they influence, or can be used to influence, the immune system of the host in many other ways. Lowimmunogenic water-soluble synthetic polymers sometimes exhibit significant immunomodulating activity, mainly concerning the activation/suppression of NK 
cells, LAK cells and macrophages. Some of them, such as poly(ethylene glycol) and poly[ $N$-(2-hydroxypropyl)methacrylamide], can be used as effective protein carriers, as they can reduce the immunogenicity of conjugated proteins and/or reduce nonspecific uptake of nanoparticle-entrapped drugs and other therapeutic agents [13].

1.3.1

Conventional Nanoparticles

Association of a cytostatic drug to colloidal carriers modifies the drug biodistribution profile, as it is mainly delivered to the mononuclear phagocytes system (liver, spleen, lungs and bone marrow). Once in the bloodstream, surface non-modified nanoparticles, (conventional nanoparticles), are rapidly opsonized and massively cleared by the fixed macrophages of the mononuclear phagocytes system organs.

The size of the colloidal carriers as well as their surface characteristics greatly influence the drug distribution pattern in the reticuloendothelial organs, since these parameters can prevent their uptake by macrophages. The exact underlying mechanism was not fully understood, but it was rapid and compatible with endocytosis. A high curvature (resulting in size $<100 \mathrm{~nm}$ ) and/or a hydrophilic surface (as opposed to the hydrophobic surface of conventional nanoparticles) are needed to reduce opsonization reactions and subsequent clearance by macrophages [14]. Since conventional nanoparticles are naturally concentrated within macrophages, they can be used to deliver drugs to these cells. The muramyl dipeptide, a lowmolecular-weight, soluble, synthetic compound based on the structure of peptidoglycan from mucobacteria, stimulates the antitumoral activity of macrophages. Although it acts on intracellular receptors, it penetrates poorly into macrophages. Furthermore, it is eliminated rapidly after intravenous administration. These problems can be overcome by encapsulation within nanocapsules, and lipophilic derivatives such as muramyl tripeptide-cholesterol have been developed to increase encapsulation efficiency. In vitro studies have shown increased intracellular penetration of muramyl peptides into macrophages when they are associated with nanoparticles, increasing macrophage effector functions (i.e., nitric oxide, cytokine and prostaglandin production) and cytostatic activity against tumor cells [3].

The contribution of conventional nanoparticles to enhance anticancer drugs efficacy is limited to targeting tumors at the organs of the mononuclear phagocytes system organ. Owing to their very short circulation time (the mean half-life of conventional nanoparticles is $3-5$ min after intravenous administration) addressing anticancer drug-loaded nanoparticles to other tumoral tissues is not reasonable. Moreover, penetration of such a carrier system across the tumoral endothelium would be minimum, leading to subtherapeutic concentrations of the drug near the cancer cells. This biodistribution can be of benefit for the chemotherapeutic treatment of mononuclear phagocytes system localized tumors (i.e., hepatocarcinoma, hepatic metastasis, bronchopulmonary tumors, myeloma and leukemia). Activity against hepatic metastases has been observed in mouse models, although this treatment is only therapeutic when the tumor load is low [15]. 
6 6 1 Conventional Chemotherapeutic Drug Nanoparticles for Cancer Treatment

Both the polymeric composition of the nanoparticles and the associated drug greatly influence the drug distribution pattern in the mononuclear phagocytic system. Conventional nanoparticles, likely, have a better safety profile than free anticancer agents when acting on normal tissue. For example, a reduction of the cardiac accumulation of drugs [16] and of the genotoxicity of mitomycin [17] have been reported. Moreover, encapsulation of doxorubicin within nanoparticles reduces its cardiotoxicity by reducing the amount of drug that reaches the myocardium with a significant increase of drug concentrations in the liver. In one study this was not associated with any overall toxicity [18] while another study, using unloaded nanoparticles, observed a reversible decline in the phagocytic capacity of the liver after prolonged dosing, as well as a slight inflammatory response [19]. Accumulation of drug nanoparticles in the liver may also influence its elimination, since this organ is the site of metabolism and biliary excretion. Biliary clearance of indomethacin was increased three-fold by inclusion in nanocapsules [20]. Nanoparticleassociated doxorubicin accumulated in bone marrow and led to a myelosuppressive effect in one study [21]. Thus, altered distribution may generate new types of toxicity. When conventional nanoparticles are used as carriers in chemotherapy, some cytotoxicity against the Kupffer cells or other targeted macrophages can be expected, as the class of drugs being used is able to induce apoptosis in these cells. Treatments featuring frequent administrations (with intervals shorter than two weeks - the restoration period of Kupffer cells) could result in a deficiency of Kupffer cells, which in turn could lead to decreased liver uptake and a subsequent decreased therapeutic efficacy for hepatic tumors. In addition, a risk for bacteriemia can not to be excluded [22]. Moreover, conventional carriers also target the bone marrow, hence chemotherapy with such carriers may increase myelosuppressive effects. However, this tropism of carriers can also be used to deliver myelostimulating compounds such as granulocyte-colony-stimulating factor [23].

Certain types of nanoparticles were also found to be able to overcome multidrug resistance mediated by the P-glycoprotein efflux system localized at the cancerous cell membrane. This simultaneous cellular resistance to multiple lipophilic drugs represents a major problem in cancer chemotherapy. Such drug resistance may appear clinically either as a lack of tumor size reduction or as the occurrence of clinical relapse after an initial positive response to antitumor treatment. The resistance mechanism can have different origins, either directly linked to specific mechanisms developed by the tumor tissue or connected to the more general problem of distribution of a drug towards its targeted tissue. Poly(alkyl cyanoacrylate) nanoparticles have been developed to overcome multidrug resistance [10]. Despite encouraging results with conventional carrier systems, much research has been dedicated to designing carriers with modified distribution and new therapeutic applications.

\section{3 .2}

\section{Sterically Stabilized Nanoparticles}

Since the usefulness of conventional nanoparticles is limited by their massive capture by the macrophages of the mononuclear phagocytes system after intravenous 
administration, systems with modified surface properties to reduce the disposition of plasma proteins and the recognition by phagocytes have been developed [14]. These are known as sterically stabilized carriers or "stealth carriers" and may remain in the blood compartment for a considerable time. The hydrophilic polymers poly(ethylene glycol), poloxamines, poloxamers, polysaccharides have been used to coat efficiently conventional nanoparticles' surface [24]. These coating provide a dynamic cloud of hydrophilic and neutral chains at the particle surface that keep away plasma proteins [25]. Poly(ethylene glycol) (PEG) has been introduced at the surface either by adsorption of surfactants [24] or by using block or branched copolymers, usually with poly(lactide) $[26,27]$. These Stealth ${ }^{\mathrm{TM}}$ nanoparticles are characterized by a prolonged half-life in the blood compartment and by extravasation into sites where the endothelium is more permeable, such as solid tumors, regions of inflammation and infection. Consequently, such long-circulating nanoparticles are supposed to be able to target directly most tumors located outside the mononuclear phagocytes system [22]. A higher tumor uptake, thanks to the small size and the hydrophilicity of the carrier device, as well as a sustained release of the drug could improve the efficacy of anticancer chemotherapy. The complementrejecting properties of nanocapsules are superior to those of nanospheres at equivalent surface area [28].

Coating conventional nanoparticles with surfactants to obtain a long-circulating carrier, the first strategy used to direct tumor targeting in vivo, has been demonstrated using photosensitizers like phthalocyanines in PEG-coated nanoparticles [29] and meta-tetra(hydroxyphenyl)chlorin in poly(lactide)-PEG nanocapsules [30].

PEG chains have been attached covalently to poly(alkyl cyanoacrylate) polymers by two chemical strategies; both types of particle have shown long-circulating properties in vivo [31]. Such particles have been loaded with tamoxifen with a view to their use in treating hormone-dependent tumors [32]. Poly(alkyl cyanoacrylate) nanoparticles allow the delivery of several drugs, including doxorubicin, across the blood-brain barrier after coating with surfactants. However, only the surfactants polysorbate (Tween 20, 40,60 and 80) and some poloxamers (Pluronic F68) can induce this uptake. A therapeutic effect with this sterically stabilized carrier system has been noted in rats bearing intracranial glioblastoma [33]. The delivery mechanism across the blood-brain barrier is most likely endocytosis via the LDL receptor by the endothelial cells lining the brain blood capillaries after injection of the nanoparticles into the blood stream. This endocytotic uptake seems to be mediated by the adsorption of apolipoprotein B and/or E adsorption from the blood. The drug, then, may be released either within these cells followed by passive diffusion into the brain or be transported into the brain by transcytosis [34]. Longcirculating nanoparticles have also been used in tumor targeting followed by irradiation of tumor site in the case of photodynamic therapy. To improve anticancer efficacy and avoid systemic phototoxicity this combination was evaluated in a EMT6 tumor-bearing mice model. Unfortunately, poly(lactic acid) nanospheres covered by PEG did not allow a higher intratumoral accumulation of its incorporated photosensitizer (hexadecafluoro zinc phthalocyanine). The fragility of the adsorbed coating and the large size $(>900 \mathrm{~nm})$ could be responsible for this failure. How- 
ever, formulation of the photosensitizer in the biodegradable nanospheres improved photodynamic therapy response, probably by influencing the intratumoral distribution pattern of the photosensitizer [35]. The biodistribution of non-biodegradable poly(methyl methacrylate) nanospheres coated or not with different surfactants (Polysorbate 80, Poloxamer 407 and Polaxamine 908) have been investigated in several mice bearing tumor models, including a murine B16-melanoma (inoculated intramuscularly), a human breast cancer MaTu (engrafted subcutaneously) and an U-373 glioblastoma (implanted intracerebrally). The coated poly(methyl methacrylate) nanospheres in circulation showed a prolonged half-life, especially with the Poloxamer 407 and Poloxamine 908 coatings. Moreover, an accumulation and retention of the coated nanospheres in the B-16 and MaTu tumors were observed, depending on the particle surface hydrophilicity and the specific growth difference of the tumor [36].

Although interesting results have been obtained with adsorbed surfactant, a covalent linkage of amphiphilic copolymers is generally preferred to obtain a protective hydrophilic cloud on nanoparticles that avoids the possibility of rapid coating desorption upon either dilution or after contact with blood components. This approach has been employed with poly(lactic acid), poly(caprolactone) and poly(cyanoacrylate) polymers, which were chemically coupled to PEG [27, 31, 37].

The surface characteristics of nanospheres prepared from poly(lactide-co-glycolide) copolymers have been optimized to reduce their interactions with plasma proteins and to increase their circulating half-life $[26,27,38]$. Biodegradable nanospheres prepared from poly(lactide-co-glycolide) coated with poly(lactide)- PEG diblock copolymers showed a significant increase in blood circulation time and reduced liver uptake in a rat model compared with non-coated poly(lactide-co-glycolide) nanospheres [39]. Nanocapsules containing poly(lactide)-PEG showed reduced association with a macrophage-like cell line (J774 A1) irrespective of dilution and incubation time up to $24 \mathrm{~h}$ [40]. This formulation, which showed a lower capture by macrophages in vitro, also gave good results in vivo, yielding a plasma area under the curve 15 -fold higher than that obtained with nanocapsules stabilized by adsorbed Poloxamer F68. Persistence in the blood compartment was accompanied by delayed liver and spleen uptake [41].

1.3.3

\section{Actively Targetable Nanoparticles}

Nanoparticles can be targeted to the particulate region of capillary endothelium, to concentrate the drug within a particular organ and allow it to diffuse from the carrier to the target tissue. The folate receptor is overexpressed on many tumor cells. Folinic acid has some advantages over transferrin or antibodies as a ligand for long-circulating carriers because it is a much smaller molecule that is unlikely to interact with opsonines and can be coupled easily to a poly(ethylene glycol) (PEG) chain without loss of receptor-binding activity. This targeting strategy has also been applied to long-circulating nanoparticles prepared from a cyanoacrylate-based polymer. Folate grafted to PEG cyanoacrylate nanoparticles has a ten-fold higher appar- 
ent affinity for the folate-binding protein than the free folate. Indeed, the particles represent a multivalent form of the ligand folic acid and folate receptors are often disposed in clusters. Thus conjugated nanoparticles could display a stronger interaction with the surface of malignant cells [42]. Bovine serum albumin nanoparticles have been reacted with the activated folic acid to conjugate folate via amino groups of the nanoparticle to improve their intracellular uptake to target cells. The nanoparticles were taken up to SKOV3 cells (human ovarian cancer cell line) and levels of binding and uptake were increased with the time of incubation until $4 \mathrm{~h}$. The levels of folate-conjugated bovine serum albumin nanoparticles were higher than those of non-conjugated nanoparticles and saturable. Association of folate conjugated bovine serum albumin nanoparticles to SKOV3 cells was inhibited by an excess amount of folic acid, suggestive of binding and/or uptake mediated by the folate receptor [43].

Folate-linked microemulsions of the antitumor antibiotic aclacinomycin have been developed and investigated both in vitro and in vivo. Three kinds of folatelinked microemulsions with different PEG chain lengths loading aclacinomycin were formulated. In vitro studies were performed in a human nasopharyngeal cell line, $\mathrm{KB}$, which overexpresses the folate receptor and in a human hepatoblastoma cell line, folate receptor (-), HepG2. In vivo experiments were carried out in a KB xenograft by systemic administration of folate-linked microemulsions loading aclacinomycin. This afforded selective folate receptor mediated cytotoxicity in KB but not in HepG2 cells. Association of the folate-PEG 5000 linked and folate-PEG 2000 linked microemulsions with the cells was 200- and 4-fold higher, respectively, whereas their cytotoxicity was 90 - and 3.5-fold higher than those of non-folate microemulsion. The folate-PEG 5000 linked microemulsions showed a 2.6-fold higher accumulation in solid tumors $24 \mathrm{~h}$ after intravenous injection and greater tumor growth inhibition than free aclacinomycin. This study showed how folate modification with a sufficiently long PEG-chain on emulsions can be an effective way of targeting emulsion to tumor cells [44].

Transferrin is another well-studied ligand for tumor targeting due to upregulation of transferrin receptors in numerous cancer cell types. A transferrin-modified, cyclodextrin polymer-based gene delivery system has been developed. The nanoparticle is surface-modified to display PEG for increasing stability in biological fluids and transferrin for targeting of cancer cells that express transferrin receptor. At low transferrin modification, the particles remain stable in physiologic salt concentrations and transfect K562 leukemia cells with increased efficiency over untargeted particles. The transferrin-modified nanoparticles are appropriate for use in the systemic delivery of nucleic acid therapeutics for metastatic cancer applications [45].

Nanoparticles conjugated with an antibody against a specific tumor antigen have been developed to obtain selective drug delivery systems for the treatment of tumors expressing a specific tumor antigen. For instance, biodegradable nanoparticles based on gelatin and human serum albumin have been developed. The surface of the nanoparticles was modified by covalent attachment of the biotin-binding protein NeutrAvidin, enabling the binding of biotinylated drug targeting ligands by 
avidin-biotin complex formation. Using the HER2 receptor specific antibody trastuzumab (Herceptin) conjugated to the surface of these nanoparticles, a specific targeting to HER2-overexpressing cells could be shown. Confocal laser scanning microscopy demonstrated an effective internalization of the nanoparticles by HER2-overexpressing cells via receptor-mediated endocytosis [46].

There is a tremendous effort to develop and test gene delivery vectors that are efficient, non-immunogenic, and applicable for cancer gene systemic therapy. Since internalization of colloidal carriers usually leads to the lysosomal compartment, in which hydrolytic enzymes will degrade both the carrier and its content, the intracellular distribution of the carrier must be modified when the encapsulated drug is a nucleic acid. Thus, systems have been developed that either fuse with the plasma membrane or have a $\mathrm{pH}$-sensitive configuration that changes conformation in the lysosomes and allows the encapsulated material to escape into the cytoplasm [3]. Rapid endo-lysosomal escape of biodegradable nanoparticles formulated from the copolymers of poly(D,L-lactide-co-glycolide) has been reported. This occurred by selective reversal of the surface charge of nanoparticles (from anionic to cationic) in the acidic endo-lysosomal compartment, which causes the nanoparticles to interact with the endo-lysosomal membrane and escape into the cytosol. These nanoparticles can deliver various therapeutic agents, including macromolecules such as DNA at a slow rate, resulting in a sustained therapeutic effect. Thermo-responsive, $\mathrm{pH}$-responsive and biodegradable nanoparticles have been developed by grafting biodegradable poly(D,L-lactide) onto N-isopropyl acrylamide and methacrylic acid. It may be sufficient for a carrier system to concentrate the drug in the tissue of interest. However, for hydrophilic molecules such as nucleic acid, which cross the plasma membrane with difficulty, intracellular delivery is required. Clinical trials for deadly pancreatic cancer have recently begun on two continents. The aim is to evaluate the safety and efficacy of engineered nanoparticles guided by a targeted delivery system to overcome dilution, filtration and inactivation encountered in the human circulatory system to deliver a killing designer gene to metastatic tumors that are refractory to conventional chemotherapy. The first patients receiving multiple intravenous infusions of the targeted delivery systemencapsulated genetic bullets have all responded favorably [47].

\subsection{4}

\section{Routes of Drug Nanoparticles Administration}

The most convenient route of drug administration is the oral one but this presents several barriers to the use of colloidal carrier owing to conditions within the gastrointestinal tract. Duodenal enzymes and bile salts destroy the lipid bilayers of most types of liposome, releasing the drug. Polymeric nanoparticles are more stable, although there is some evidence that polyesters can be degraded by pancreatic lipases [48]. They may be able to improve bioavailability, particularly for highly insoluble drugs, by increasing the surface area for dissolution and as a result of bioadhesion. However, nanoparticles can be used to protect a labile drug from degradation in the gastrointestinal tract or to protect it from toxicity due to the drug. 
Polymeric nanoparticles, due to their bioadhesive properties, may be immobilized within the mucus or, when in contact with the epithelial cells, show a slower clearance from the gastrointestinal tract [49]. Nanoparticles of biodegradable polymers containing alpha-tocopheryl PEG 1000 succinate (vitamin E TPGS) have been proposed to replace the current method of clinical administration and to provide an innovative solution for oral chemotherapy. Vitamin E TPGS could be a novel surfactant as well as a matrix material when blended with other biodegradable polymers - it has great advantages for the manufacture of polymeric nanoparticles for controlled release of paclitaxel and other anticancer drugs [50].

Potential applications of colloidal drug carriers by the intravenous route can be summarized as concentrating drugs in accessible sites, rerouting drugs away from sites of toxicity and increasing the circulation time of labile or rapidly eliminated drugs.

After subcutaneous or intraperitoneal administration, nanoparticles are taken up by regional lymph nodes [51].

Subcutaneously or locally injected (in the peri-tumoral region) nanoparticles can be used for lymphatic targeting as a tool for chemotherapy against lymphatic tumors or metastases since they penetrate the interstitial space around the injection site and are gradually absorbed by the lymphatic capillaries into the lymphatic system. Aclarubicin adsorbed onto activated carbon particles has been tested after subcutaneous injection in mice, against a murine model (P288 leukemia cells) of lymph node metastases [52]. The same system has also been used as a locoregional chemotherapy adjuvant for breast cancer in patients after intratumoral and peritumoral injections [53]. In both cases, this carrier system distributed selectively high levels of free aclarubicin to the regional lymphatic system and low levels to the rest of the body. However, this carrier system is not biodegradable and it is rather big $(>100 \mathrm{~nm})$, impeding drainage from the injection site. In addition, the drug is associated to the particles by adsorption, leading to a rapid release with possible systemic absorption. Biodegradable systems coated by adsorption of the surfactant Poloxamine 904 or poly(isobutyl cyanoacrylate) nanocapsules [51, 54] have been developed to improve lymphatic targeting. Poloxamine 904 caused an increased sequestration of the particles in lymph nodes, reducing the systemic absorption of any encapsulated drug [54]. Poly(isobutyl cyanoacrylate) nanocapsules were also able to retain the lipophilic indicator 12-(9-anthroxy)stearic acid in the regional lymph nodes for $168 \mathrm{~h}$ after intramuscular administration [51]. A study combining intratumoral administration of gadolinium-loaded chitosan nanoparticles and neutron-capture therapy has been performed on the B16F10 melanoma model subcutaneously implanted in mice. Gadolinium retention in tumor tissue increased when it was encapsulated with respect to the free drug. Tumor irradiation $8 \mathrm{~h}$ after the last intratumoral injection of gadolinium nanoparticles prevented further tumor growth in the animals treated, thereby increasing their life expectancy [55].

Retention of carriers instilled into the eye also occurs, leading to important therapeutic potential in this area. As well as a bioadhesive effect, some evidence has been presented to show that nanoparticles can penetrate through the corneal epithelium [56]. 
12 1 Conventional Chemotherapeutic Drug Nanoparticles for Cancer Treatment

1.4

Anticancer Drug Nanoparticles

1.4.1

Anthracyclines

One of the most powerful and widely used anticancer drugs is doxorubicin, an anthracyclinic antibiotic that inhibits the synthesis of nucleic acids. This drug has a very narrow therapeutic index as its clinical use is hampered by several undesirable side-effects like cardiotoxicity and myelosuppression [57]. Thus, much effort has been made to target doxorubicin to cancer tissues, improving its efficacy and safety.

Poly(isohexyl cyanoacrylate) nanospheres incorporating doxorubicin have been developed. On an hepatic metastases model in mice (M5076 reticulum cell sarcoma) such doxorubicin nanospheres afforded a greater reduction in the number of metastases than when free doxorubicin was used and it appeared to increase the life span of the metastasis-bearing mice [58]. Another study found higher concentrations of doxorubicin in mice liver, spleen and lungs with doxorubicin incorporated into poly(isohexyl cyanoacrylate) than in mice treated with free doxorubicin. At the same time, the concentration of doxorubicin in heart and kidneys of the mice were lower than when free doxorubicin was used [18]. Tissue pharmacokinetic studies showed that the underlying mechanism responsible for the increased therapeutic efficacy of the nanoparticle formulation was a transfer of doxorubicin from the healthy hepatic tissue, acting as a drug reservoir, to the malignant cells. Kupffer cells, after a massive uptake of nanoparticles by phagocytosis, were able to induce the release of doxorubicin, leading to a gradient concentration, favorable for a prolonged diffusion of the free and still active drug towards the neighboring metastatic cells [59]. Thus, this biodistribution profile can be of benefit for chemotherapeutic treatment of tumors localized in the mononuclear phagocytic system.

Clinical pharmacokinetics after a single intravenous administration of doxorubicin adsorbed onto poly(methacrylate) nanospheres has been investigated in hepatoma patients. This type of conventional carrier, although of limited use in vivo because not biodegradable, allowed a reduction in both the volume of distribution and the elimination half-life of doxorubicin [60]. Another phase I clinical investigation has been carried out on 21 cancer patients with doxorubicin associated to biodegradable poly(isohexyl cyanoacrylate) nanospheres. Pharmacokinetic studies conducted in 3/21 patients revealed important interindividual variation for doxorubicin (encapsulated or not) plasma levels. Clinical toxicity of encapsulated doxorubicin consisted of dose-dependent myelosuppression of different grades in all patients, in pseudo-allergic reactions in $3 / 21$ patients and in diffuse bone pain in $3 /$ 21 patients. However, neither cardiac toxicity nor hepatotoxicity were encountered among 18 patients treated with the nanoparticles formulation. According to WHO criteria, there were only $2 / 21$ stable diseases lasting 4-6 months. All the other patients had progressive disease after the first course of doxorubicin-loaded poly(isohexyl cyanoacrylate) nanospheres. This could be due to tumor localization, as 
they were rarely located at sites of the mononuclear phagocytic system, resulting in subtherapeutic anticancer drug concentration exposure [61].

As doxorubicin-loaded poly(isobutyl cyanoacrylate) nanoparticles are more efficient than free drug in mice bearing hepatic metastasis of the M5076 tumor, and Kupffer cells could have acted as a drug reservoir after nanoparticle phagocytosis, the role of macrophages in mediating the cytotoxicity of doxorubicin-loaded nanoparticles on M5076 cells was studied. After direct contact, free doxorubicin and doxorubicin-loaded nanoparticles had the same efficacy against M5076 cell growth. Co-culture experiments with macrophages J774.A1 led to a five-fold increase in the $\mathrm{IC}_{50}$ for both doxorubicin and doxorubicin-loaded nanoparticles. The activation of macrophages by IFN- $\gamma$ in co-culture significantly decreased the $\mathrm{IC}_{50} \mathrm{~s}$. After phagocytosis of doxorubicin-loaded nanoparticles, J774.A1 cells were able to release active drug, allowing it to exert its cytotoxicity against M5076 cells. Drug efficacy was potentiated by the activation of macrophages releasing cytotoxic factors such as NO, which resulted in increased tumor cell death [62]. Similarly, the use in a murine tumor model (implanted subcutaneously J774A.1 macrophages) of a dextrandoxorubicin conjugate incorporated into small chitosan nanospheres was reported to outperform the free conjugate, especially in relation to life expectancy [63].

The body distribution of nanoparticle incorporating doxorubicin injected into the hepatic artery of hepatoma bearing rats was investigated. The nanoparticle formulation and free doxorubicin were injected into the hepatic artery of Walker-256 hepatoma bearing rats on the seventh day after tumor implantation. Survival time, tumor enlargement ratio and tumor necrosis degree were compared. Nanoparticles containing doxorubicin substantially increased the drug concentrations in liver, spleen, and tumor of rats compared to free drug, whereas the concentrations in plasma, heart and lungs were significantly decreased. The nanoparticulate formulation brought on a more significant tumor inhibition and more extensive tumor necrosis. The prolonged life span ratio was $109.22 \%$ as compared with rats that accepted normal saline $[64,65]$.

The nanoparticle modified biodistribution may generate new types of toxicity, as has been observed with doxorubicin incorporated in poly(isobutyl cyanoacrylate) and poly(isohexyl cyanoacrylate) nanospheres, whose hematopoietic toxicity was generally more pronounced and long-lasting than that of free doxorubicin [21]. Acute renal toxicity was another murine-reported doxorubicin toxicity, which was amplified by the association of the drug to poly(isobutyl cyanoacrylate) nanospheres. Proteinuria was probably the result of a modified biodistribution of the associated drug, which resulted in glomerular damage [66]. In another instance, doxorubicin was incorporated into gelatin nanospheres by a covalent bond on its amino group via glutaraldehyde [67]. Unfortunately, no or only marginal antitumor activity against a C26 tumor (mouse colon adenocarcinoma) in vivo was showed, and in certain cases there was even an increased doxorubicin cardiotoxicity. Once again this lack of antitumor activity may due to slow dissociation of the complex due to the covalent linkage or to the slow diffusion of the complex across the cellular membrane.

Polysorbate 80-coated poly(isobutyl cyanoacrylate) nanospheres provided no ma- 
jor changes in doxorubicin biodistribution and pharmacokinetic parameters in rats when compared to those of conventional poly(isobutyl cyanoacrylate) nanospheres. However, the coated nanospheres did transport a significant amount of incorporated doxorubicin to the brain of healthy rats, reaching the highest drug levels in this organ $2-4 \mathrm{~h}$ after intravenous administration. Consequently, an active transport from the blood to the brain was suspected since cerebral accumulation occurred against a concentration gradient. A mechanism involving an endocytosis by brain endothelial cells was hypothesized, as the brain uptake was inhibited at $4{ }^{\circ} \mathrm{C}$ and after a pretreatment with cytochalasin B [68]. Then, polysorbate 80-coated poly(isobutyl cyanoacrylate) nanoparticles were shown to enable the transport of doxorubicin across the blood-brain barrier to the brain after intravenous administration and to considerably reduce the growth of brain tumors in rats. The acute toxicity of doxorubicin associated with polysorbate 80-coated nanoparticles in healthy rats was studied and a therapeutic dose range for this formulation in rats with intracranially implanted 101/8 glioblastoma was established. Single intravenous administration of empty poly(isobutyl cyanoacrylate) nanoparticles in the dose range $100-400 \mathrm{mg} \mathrm{kg}^{-1}$ did not cause mortality within the period of observation. Association of doxorubicin with poly(isobutyl cyanoacrylate) nanoparticles did not produce significant changes of quantitative parameters of acute toxicity of the antitumor agent. Likewise, the presence of polysorbate 80 in the formulations was not associated with changes in toxicity compared with free or nanoparticulate drug. The results in tumor-bearing rats were similar to those in healthy rats. The toxicity of doxorubicin bound to nanoparticles was similar or even lower than that of free doxorubicin [69]. As doxorubicin bound to polysorbate-coated nanoparticles crossed the intact blood-brain barrier, reaching therapeutic concentrations in the brain, the therapeutic potential of this formulation in vivo was studied using an animal model created by implantation of $101 / 8$ glioblastoma tumor in rat brains. Rats treated with doxorubicin bound to polysorbate-coated nanoparticles had significantly higher survival times than with free doxorubicin; over $20 \%$ of the animals showed a long-term remission (Fig. 1.1). All animals treated with polysorbate-containing formulation also had a slight inflammatory reaction to the tumor. There was no indication of neurotoxicity [70].

Doxorubicin has also been conjugated chemically to a terminal end group of poly(D,L-lactic-co-glycolic acid) by an ester linkage and then formulated into nanoparticles. The conjugated doxorubicin nanoparticles showed increased uptake within a HepG2 cell line. They exhibited a slightly lower $\mathrm{IC}_{50}$ against the HepG2 cell line than did free doxorubicin. An in vivo antitumor activity assay also showed that a single injection of the nanoparticles had comparable activity to that of free doxorubicin administered by daily injection [71]. Doxorubicin-loaded poly(butyl cyanoacrylate) nanoparticles have been used to enhance the delivery of the drug to Dalton's lymphoma solid tumor. ${ }^{99 \mathrm{~m}}$ Tc-labeled complexes of doxorubicin and doxorubicin-loaded poly(butyl cyanoacrylate) nanoparticles were administered subcutaneously below the Dalton's lymphoma tumor. The distribution of doxorubicinloaded poly(butyl cyanoacrylate) nanoparticles to the blood, heart and organs of the 




Fig. 1.1. Kaplan-Meier survival curves. Percentage of survival of the rats with intracranially transplanted glioblastoma after intravenous injection of $2.5 \mathrm{mg} \mathrm{kg}^{-1}$ doxorubicin each on day 2, 5 and 8 using one of the following formulations: DOX, doxorubicin in saline; DOX+Ps, doxorubicin

\author{
in saline plus polysorbate 80; DOX-NP, \\ doxorubicin bound to poly(butyl cyanoacrylate) \\ nanoparticles; DOX-NP+Ps, doxorubicin \\ bound to poly(butyl cyanoacrylate) \\ nanoparticles coated with polysorbate 80 . \\ (Reproduced with permission from Ref. [70].)
}

reticuloendothelial system was biphasic with a rapid initial distribution, followed by a significant decrease later at $6 \mathrm{~h}$ post-injection. The distribution of doxorubicin to tissues was very low initially and increased significantly at $6 \mathrm{~h}$ post-injection, indicating its accumulation at the injection site for a longer time. The concentration of doxorubicin-loaded poly(butyl cyanoacrylate) nanoparticles was also high in tissues at $6 \mathrm{~h}$ post-injection, indicating their accumulation at the subcutaneous site and consequent disposition to tissues with time. A significantly high tumor uptake of doxorubicin-loaded poly(butyl cyanoacrylate) nanoparticles (approx. 13-fold higher at $48 \mathrm{~h}$ post-injection) was found compared to free doxorubicin. Tumor concentrations of both doxorubicin and doxorubicin-loaded poly(butyl cyanoacrylate) nanoparticles increased with time, indicating their slow penetration from the injection site into tumor [72].

Poly(ethylene glycol)-coated poly(hexadecyl cyanoacrylate) nanospheres also displayed a significant accumulation within an orthotopic 9L gliosarcoma model, after intravenous administration to rats. Hence, in the same model, the pre-clinical efficacy of this carrier when loaded with doxorubicin was evaluated. The cumulative maximum tolerated dose of nanoparticulate doxorubicin was 1.5-fold higher than that of free doxorubicin. Nevertheless, encapsulated doxorubicin was unable to elicit a better therapeutic response in the 9L gliosarcoma. A biodistribution study revealed that the doxorubicin-loaded nanospheres accumulated to a 2.5 -fold lesser extent in the 9L tumor than did the unloaded nanospheres and that they were mainly 
localized in the lungs and the spleen. Such a typical profile indicated aggregation with plasma proteins as a consequence of the positive surface charge of these loaded particles; this ionic interaction resulting from drug encapsulation was mainly responsible for 9L treatment failure [73].

The ability of doxorubicin-loaded solid lipid nanoparticles to achieve prolonged drug plasma levels was also investigated. One study observed a low uptake of solid lipid nanoparticles by the liver and spleen macrophages. This might be explained by a low surface hydrophobicity of the nanoparticles avoiding the adsorption of any blood proteins mediating the uptake by liver and spleen macrophages [74]. Moreover, uptake of the solid lipid nanoparticles (SLNs) by the brain might be explained by adsorption of a blood protein mediating adherence to the endothelial cells of the blood-brain barrier [75]. Pharmacokinetic studies of doxorubicin incorporated into SLNs showed higher blood levels in comparison to a commercial drug solution after intravenous injection in rats. Concerning the body distribution, doxorubicinloaded SLNs caused higher drug concentrations in lung, spleen and brain, while the solution led to a distribution more into liver and kidneys [76]. Furthermore, incorporation of doxorubicin into SLNs strongly enhanced its cytotoxicity in several cell lines [77, 78]. In particular, in the human colorectal cancer cell line HT-29, the intracellular doxorubicin content was double after $24 \mathrm{~h}$ exposure to loaded SLNs versus the conventional drug formulation [78]. Pharmacokinetics and tissue distribution of doxorubicin incorporated in non-sterically stabilized SLNs and in sterically stabilized SLNs at increasing concentration of stearic acid-PEG 2000 as stabilizing agent after intravenous administration to rabbits have been studied. The doxorubicin area under the concentration-time curve increased as a function of the amount of sterically stabilizing agent present in the SLNs. Doxorubicin was present in the brain only after doxorubicin-loaded SLNs administration and the increase in the stabilizing agent affected the doxorubicin transported into the brain. There was always less doxorubicin in the liver, lungs, spleen, heart and kidneys after injection of any of the types of SLNs than after the doxorubicin solution. In particular, all SLNs formulations significantly decreased heart and liver concentrations of doxorubicin [79]. Interestingly, both non-coated and coated SLNs showed a similar low uptake by liver and spleen macrophages [80]. The bioavailability of idarubicin can be also improved by administering idarubicin-loaded SLNs duodenally to rats. The pharmacokinetic parameters of idarubicin found after duodenal administration of idarubicin solution and idarubicin-loaded SLNs were different. The area under the concentration-time curve and the elimination half-life were 2- and 30fold higher after administration of idarubicin-loaded SLNs than after solution administration, respectively. Tissue distribution also differed: idarubicin and its main metabolite idarubicinol concentrations were lower in heart, lung, spleen and kidneys after idarubicin-loaded SLNs administration than after solution administration. Furthermore, the drug and its metabolite were detected in the brain only after idarubicin-loaded SLNs administration, indicating that SLNs were able to cross the blood-brain barrier. After intravenous idarubicin-loaded SLNs administration, the area under the time-concentration curve of idarubicin was lower than after duodenal administration of the same formulation [81]. 
1.4.1.1 Reverse of P-glycoprotein Mediated Multidrug Resistance of Cancer Cells to Doxorubicin

Different types of nanoparticles have been developed to reverse the P-glycoprotein mediated multidrug resistance of cancer cells to doxorubicin, an important problem in its clinical use.

Conventional poly(cyanoacrylate) nanoparticles allow doxorubicin P-glycoprotein mediated multidrug resistance to be overcome in vitro only when the nanoparticles and the resistant cancer cell line are in close contact [82]. The nanoparticleassociated drug accumulated within the cells and appeared to avoid P-glycoprotein dependent efflux. This reversal was only observed with poly(alkyl cyanoacrylate) nanoparticles and was not due to particle endocytosis. The formation of a complex between positively charged doxorubicin and negatively charged polymer degradation products seemed to favor diffusion across the plasma membrane [82].

When doxorubicin was coupled via an ionic interaction to non-biodegradable poly(methacrylate) nanospheres, the P-glycoprotein mediated multidrug resistance reversal differed from that of doxorubicin incorporated into biodegradable poly(cyanoacrylate) nanoparticles.

When adsorbed onto the surface of poly(methacrylate) nanospheres, doxorubicin was demonstrated to be cell internalized by an endocytic process in cultured rat hepatocytes and in a human monocyte-like cancer cell line expressing P-glycoprotein (U-937). Once internalized, poly(methacrylate) nanospheres generated an intracellular sustained release of doxorubicin in U-937 cells. A higher intracellular accumulation, related to a more important cytotoxicity on U-937 cells, was noted for encapsulated doxorubicin than for free doxorubicin. However, such a carrier, despite its ability to mask the positive charge of doxorubicin, is of limited use in vivo since it is not biodegradable [83].

When doxorubicin was incorporated into rapidly biodegraded poly(isobutyl cyanoacrylate) nanospheres and tested in a resistant murine leukemia sub-line overexpressing P-glycoprotein (P388/ADR), a higher cellular uptake was observed. Furthermore, the cell uptake kinetics of doxorubicin nanoparticles were unchanged in the presence of cytochalasin B, an endocytosis inhibitor. Efflux studies showed a similar profile for doxorubicin in nanoparticulate or free form. This suggests that poly(isobutyl cyanoacrylate) nanospheres did not enter the cells. In an in vitro model of doxorubicin-resistant rat glioblastoma (C6 cells sub-lines), doxorubicin incorporated into poly(isobutyl cyanoacrylate) nanospheres was always more cytotoxic, and also had a lower intracellular concentration, than the free drug. The polymer constituting the nanospheres did not inhibit the P-glycoprotein by direct interaction with the protein. It was also observed that on $\mathrm{C} 6$ cell sub-lines with different expression of P-glycoprotein that doxorubicin nanospheres were only efficient on pure P-glycoprotein mediated multidrug resistance phenotype cells and not on the additional mechanisms of resistance to doxorubicin [84]. Thus, the mechanism of P-gp reversion by nanoparticles could only be explained by a local delivery of the drug in high concentration close to the cell membrane, after degradation of the polymeric carrier. Such local microconcentration of doxorubicin was supposed to be able to saturate P-glycoprotein [85]. P-glycoprotein mediated multidrug resis- 
tance is overcome with poly(isobutyl cyanoacrylate) and poly(isobutyl cyanoacrylate) doxorubicin-loaded nanospheres not only due to the adsorption of nanoparticles to the cell surface but also due to an increased diffusion of doxorubicin across the plasma membrane, thanks to the formation of an ion pair between negatively charged cyanoacrylic acid (a nanoparticle degradation product) and the positively charged doxorubicin. Such ion-pair formation has been evidenced by Raman spectroscopy and by ion-pair reversed-phase HPLC [86]. Masking the positive charge of the amino sugar of doxorubicin appears to be key to overcoming P-gp mediated multidrug resistance. At the same time, the cytotoxic activity of doxorubicin was only slowly compromised after chemical modifications of the amino sugar. Consequently, some studies focused on developing systems featuring covalent linkage between the polymers and the amino sugar of doxorubicin [87]. Such a complex mechanism for overcoming P-glycoprotein mediated multidrug resistance was only observed with cyanoacrylate nanoparticles.

The failure to overcome P-glycoprotein mediated multidrug resistance with nanoparticles designed with other polymers could be explained by an appropriate release mechanism of drug (diffusion could lead to the release of the active compound without the polymeric counter ion), by degradation kinetics of the polymer that are too slow, or by the size of the polymeric counter ion, which could be the limiting factor for diffusion across the cell membrane.

Interestingly, the association of doxorubicin with poly(alkyl cyanoacrylate) nanoparticles also reversed the resistance to doxorubicin in numerous multidrugresistant cell lines [88].

Furthermore, doxorubicin encapsulated in poly(isohexyl cyanoacrylate) nanospheres can circumvent P-glycoprotein mediated multidrug resistance. K562 and MCF7 cell lines were more resistant to free doxorubicin than to doxorubicin poly(isohexyl cyanoacrylate) nanospheres. The MCF7 sub-lines selected with doxorubicin poly(isohexyl cyanoacrylate) nanospheres exhibited a higher level of resistance to both doxorubicin formulations than those selected with free doxorubicin. Different levels of overexpression of several genes involved in drug resistance occurred in the resistant variants. MDR1 gene overexpression was consistently higher in free doxorubicin selected cells than in doxorubicin poly(isohexyl cyanoacrylate) nanosphere selected cells, while this was the reverse for the BCRP gene. Overexpression of the MRP1 and TOP2 alpha genes was also observed in the selected variants. Thus, drug encapsulation markedly alters or delays the several mechanisms involved in the acquisition of drug resistance [89].

Other strategies to bypass doxorubicin multidrug resistance, such as the use of Stealth ${ }^{\mathrm{TM}}$ poly(cyanoacrylate) nanoparticles, could be considered, as well as the co-administration of doxorubicin with chemo-sensitizing agents, generally acting as P-glycoprotein inhibitors. Co-encapsulation of the reversing agent cyclosporin and doxorubicin into poly(isobutyl cyanoacrylate) nanospheres was investigated to reduce the side-effects of both drugs while enhancing their efficacy. This formulation, compared to incubation of cyclosporin and doxorubicin, or doxorubicin nanoparticles and cyclosporin, elicited the most effective growth rate inhibition on 
P388/ADR cells. Such a high efficacy was supposed to result from the synergistic effect of the rapid release of both doxorubicin and cyclosporin at the surface of cancer cells, allowing a better internalization of doxorubicin, while inhibiting its efflux by blocking the P-gp with the cyclosporin [90].

1.4.2

Antiestrogens

Nanospheres and nanocapsules made of biodegradable copolymers and coated with poly(ethylene glycol) (PEG) chains have been developed as parenteral delivery system for the administration of the anti-estrogen 4-hydroxytamoxifen RU 58668 (RU). Coating with PEG chains lengthened the anti-estrogen activity of RU, with prolonged antiuterotrophic activity of the encapsulated drug into PEG-poly(D,Llactic acid) nanospheres as compared with non-coated nanospheres. In mice bearing MCF-7 estrogen-dependent tumors, free RU injected by the intravenous route slightly decreased estradiol-promoted tumor growth while RU-loaded PEG-poly(D,L-lactic acid) nanospheres injected at the same dose strongly reduced it. The antitumoral activity of RU encapsulated within PEGylated nanocapsules was stronger than that of RU entrapped with PEGylated nanospheres loaded at an equivalent dose. The PEGylated nanocapsules decreased the tumor size in nude mice transplanted with estrogen receptor-positive but estrogen-independent MCF-7/Ras breast cancer cells at a concentration 2.5-fold lower than that of the PEGylated nanospheres [91, 92].

To increase the local concentration of tamoxifen in estrogen receptor-positive breast cancer cells poly( $\varepsilon$-caprolactone) nanoparticle formulation has been developed. Poly( $\varepsilon$-caprolactone) nanoparticles labeled with rhodamine123 were incubated with MCF-7 estrogen receptor-positive breast cancer cells. A significant fraction of the administered rhodamine123-loaded poly( $\varepsilon$-caprolactone) nanoparticles was found in the perinuclear region of the MCF-7 cells, where estrogen receptors are also localized, after $1 \mathrm{~h}$ of incubation. These nanoparticles were rapidly internalized in MCF-7 cells and intracellular tamoxifen concentrations followed a saturable process [93]. In another study, the biodistribution profile of tamoxifen encapsulated in polymeric nanoparticulate formulations after intravenous administration was evaluated, with or without surface-stabilizing agents. In vivo biodistribution studies of tamoxifen-loaded poly(ethylene oxide)-modified poly( $\varepsilon$ caprolactone) nanoparticles were carried out in $\mathrm{Nu} / \mathrm{Nu}$ athymic mice bearing a human breast carcinoma xenograft, MDA-MB-231, using tritiated $\left[{ }^{3} \mathrm{H}\right]$ tamoxifen as radio-marker for quantification. After intravenous administration the drugloaded nanoparticles accumulated primarily in the liver, though up to $26 \%$ of the total activity could be recovered in tumor at $6 \mathrm{~h}$ post-injection for poly(ethylene oxide)-modified poly( $\varepsilon$-caprolactone) nanoparticles. In comparison with free drug and uncoated nanoparticles, the modified nanoparticles exhibited a significantly increased level of accumulation of the drug within the tumor with time as well as prolonged drug presence in the systemic circulation [94]. 
1.4 .3

Anti-metabolites

Poly(amidoamine) dendritic polymers coated with poly(ethylene glycol) have been developed to deliver 5-fluorouracil. In rats after intravenous administration this nanoparticle formulation showed a lower drug clearance than after the free drug administration [95]. In addition, poly(D,L-lactide)-g-poly( $N$-isopropyl acrylamide-comethacrylic acid) nanoparticles have been studied as drug carrier for intracellular delivery of 5-fluorouracil [96].

Methotrexate has been incorporated in modified poly(amidoamine) dendritic polymers conjugated to folic acid as a targeting agent. These conjugates were injected intravenously into immunodeficient mice bearing human KB tumors that overexpress the folic acid receptor. Targeting methotrexate increased its antitumor activity and markedly decreased its toxicity, allowing therapeutic responses not possible with the free drug [97].

1.4.4

Camptothecins

Solid lipid nanoparticles (SLNs) are a promising sustained release system for camptothecin after oral administration. The pharmacokinetics and body distribution of camptothecin after intravenous injection in mice have been studied. Two plasma peaks were observed after administration of camptothecin-loaded SLNs. The first was attributed to the presence of free drug, the second peak can be attributed to controlled release or potential gut uptake of the SLNs. In comparison to the drug solution, SLNs lead to a much higher area under the concentration-time curve/dose and mean residence times, especially in brain, heart and organs containing reticuloendothelial cells. The highest area under the concentration time curve ratio of SLNs to drug solution among the tested organs was found in the brain. Incorporation of camptothecin into SLNs also prevented its hydrolysis [98].

The in vitro and in vivo antitumor characteristics of methoxy poly(ethylene glycol)-poly(D,L-lactic acid) nanoparticles containing camptothecin have been examined. After intravenous administration in rats, camptothecin-loaded nanoparticles showed a longer plasma retention than camptothecin solution and high and long tumor localization. In both single and double administration to mice bearing sarcoma 180 solid tumor, camptothecin-loaded nanoparticles were much more effective than camptothecin solution, in particular the tumor disappeared completely in three of the four mice after double administration of camptothecin-loaded nanoparticles [99].

Irinotecan-containing nanoparticles have been prepared by co-precipitation with addition of water to an acetone solution of poly(D,L-lactic acid), PEG-blockpoly(propylene glycol)-block-PEG and irinotecan. When the antitumor effect was examined using mice bearing sarcoma 180 subcutaneously, only nanoparticles suppressed tumor growth significantly. After intravenous injection in rats, nanopar- 
ticles maintained irinotecan plasma concentration longer than irinotecan aqueous solution [100].

Lipid based nanoparticles incorporating the irinotecan analog $\mathrm{SN}-38$ have been developed and studied in vitro and in vivo. Interestingly, incorporation of SN-38 into nanoparticles improved the stability of the active drug, the lactone form, in serum-containing medium. Furthermore, studies in nude mice showed a prolonged half-life of the active drug in whole blood and increased efficacy compared to irinotecan in a mouse xenograft tumor model [101].

1.4 .5

Cisplatin

Nanoparticles prepared from poly(lactide-co-glycolide) copolymers increase the circulating half-life of cisplatin [102]. A system for the local delivery of chemotherapy to malignant solid tumors has been developed based on calcium phosphate nanoparticles containing cisplatin. Cytotoxicity was investigated in a K8 clonal murine osteosarcoma cell line. Drug activity was retained after adsorption onto the apatite crystals and the apatite/cisplatin formulation exhibited cytotoxic effects with a dose-dependent decrease of cell viability [103].

1.4.6

Paclitaxel

Paclitaxel, a microtubule-stabilizing agent that promotes polymerization of tubulin causing cell death by disrupting the dynamics necessary for cell division, is effective against a wide spectrum of cancers, including ovarian cancer, breast cancer, small and non-small cell lung cancer, colon cancer, head and neck cancer, multiple myeloma, melanoma and Kaposi's sarcoma. In clinical practice high incidences of adverse reactions of the drug such as neurotoxicity, myelosuppression and allergic reactions have been reported. Since its clinical administration is hampered by its poor solubility in water, excipients such as Cremophor EL (polyethoxylated castor oil) and ethanol are used in the pharmaceutical drug formulation of the current clinical administration [104].

Cremophor EL and polysorbate 80 (Tween 80 ) are widely used as drug formulation vehicles for the taxane anticancer agents paclitaxel and docetaxel. Both solubilizers are biologically and pharmacologically active compounds, and their use as drug formulation vehicles has been implicated in clinically important adverse effects, including acute hypersensitivity reactions and peripheral neuropathy. Cremophor EL and Tween 80 have also been demonstrated to influence the disposition of solubilized drugs that are administered intravenously. The overall resulting effect is a highly increased systemic drug exposure and a simultaneously decreased clearance, leading to alteration in the pharmacodynamic characteristics of the solubilized drug. Kinetic experiments revealed that this effect is caused primarily by reduced cellular uptake of the drug from large spherical micellar-like structures with a highly hydrophobic interior, which act as the principal carrier of circulating drug. 
The existence of Cremophor EL and Tween 80 in blood as large polar micelles has also raised additional complexities in the case of combination chemotherapy regimens with taxanes, such that the disposition of several co-administered drugs, including anthracyclines and epipodophyllotoxins, is significantly altered. In contrast to the enhancing effects of Tween 80, addition of Cremophor EL to the formulation of oral drug preparations seems to result in significantly diminished drug uptake and reduced circulating concentrations [105]. Nanoparticles of biodegradable polymers can provide an ideal solution to such an adjuvant problem and realize a controlled and targeted delivery of paclitaxel with better efficacy and less side-effects. With further development, such as particle size optimization and surface coating, nanoparticle formulation of paclitaxel can promote full paclitaxel efficacy, thereby improving the quality of life of the patients.

Paclitaxel incorporated into poly(vinylpyrrolidone) nanospheres has been assayed on a B16F10 murine melanoma transplanted subcutaneously in C57B1/6 mice. Mice treated with repeated intravenous injections of paclitaxel-loaded nanospheres showed a significant tumor regression and higher survival rates than mice treated with free paclitaxel [106]. Sterically stabilized SLNs were also prepared to prolong the blood circulation time following intravenous administration [107]. Incorporation of paclitaxel into SLNs enhanced paclitaxel cytotoxicity on the human breast adenocarcinoma cell line MCF-7, but not on the human promyelocytic leukemia cell line HL-60 [77].

In a phase I study, ABI-007 - a novel formulation prepared by high-pressure homogenization of paclitaxel in the presence of human serum albumin, which results in a nanoparticle colloidal suspension - was found to offer several clinical advantages, including a rapid infusion rate and a high maximum tolerated dose. Furthermore, the absence of Cremophor EL allowed ABI-007 to be administered to patients without a need for premedication that is routinely used to prevent the hypersensitivity reactions associated with the conventional formulation of paclitaxel. Hematologic toxicities were mild throughout treatment, while most nonhematologic toxicities were grade 1 or 2 [108].

The feasibility, maximum tolerated dose, and toxicities of intraarterial administration of ABI-007 were studied in patients with advanced head and neck and recurrent anal canal squamous cell carcinoma in 43 patients ( 31 with advanced head and neck and 12 with recurrent anal canal squamous cell carcinoma). Patients were treated intraarterially with paclitaxel-albumin nanoparticles every four weeks for three cycles. Paclitaxel albumin nanoparticles were compared preliminarily with paclitaxel for in vitro cytostatic activity. Significantly, pharmacokinetic profiles after intraarterial administration were obtained (Fig. 1.2). The doselimiting toxicity of the nanoparticles formulation was myelosuppression consisting of neutropenia in three patients. Non-hematologic toxicities included total alopecia, gastrointestinal toxicity, skin toxicity, neurologic toxicity, ocular toxicity, flu-like. The maximum tolerated dose in a single administration was $270 \mathrm{mg} \mathrm{m}^{-2}$. Most dose levels showed considerable antitumor activity $(80.9 \%$ complete response and partial response) [109].

The effectiveness of intraarterial infusion of paclitaxel incorporated into human 

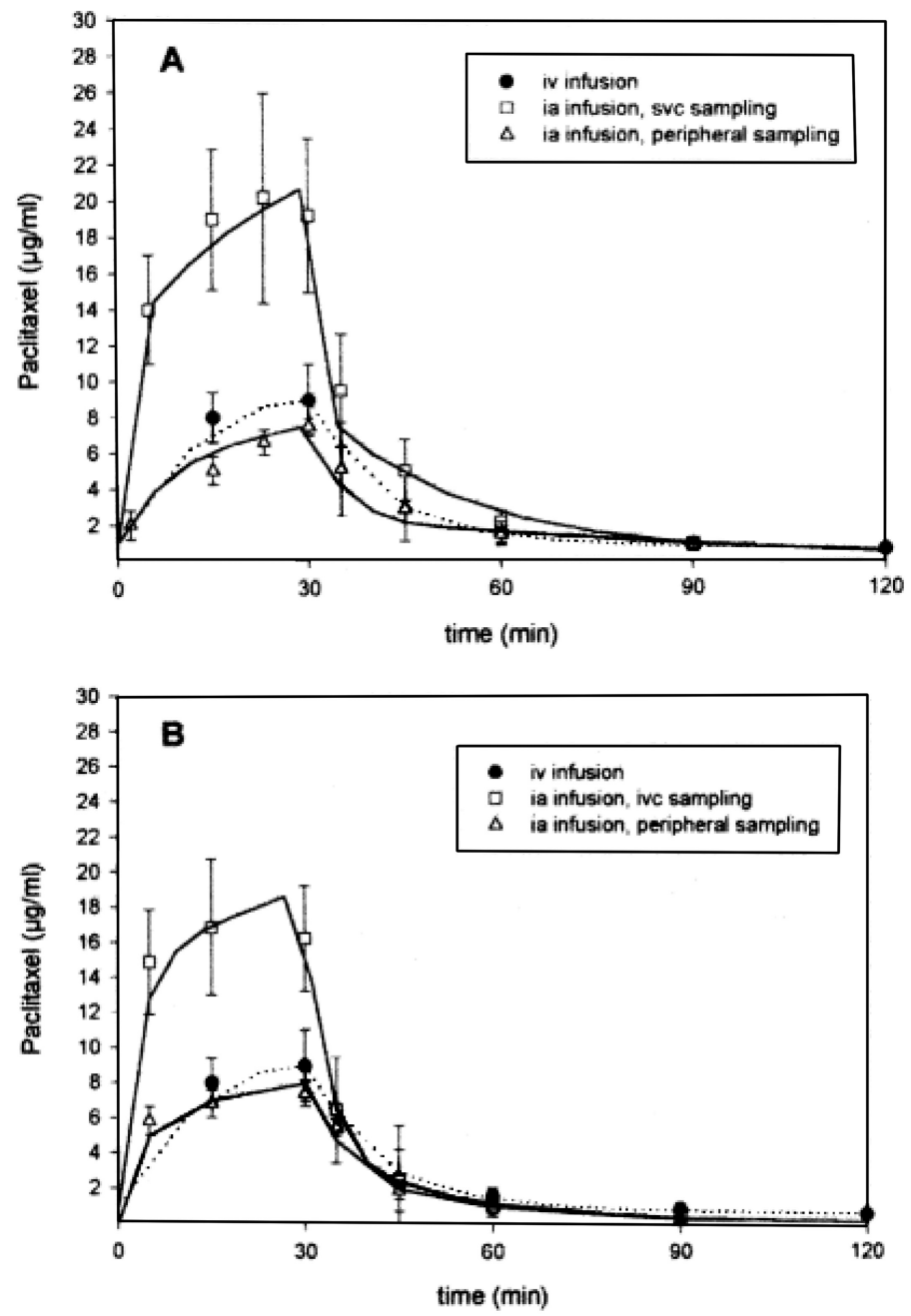

Fig. 1.2. Concentration-time curves. Mean paclitaxel concentration versus time profiles in patients with head and neck $(A)$ or anal canal

constant infusion of ABI-007 $\left(250 \mathrm{mg} \mathrm{m}^{-2}\right.$ of (B) carcinomas during and after $30 \mathrm{~min}$ paclitaxel); iv, intravenous; ia, intraarterial; svc, superior vena cava; ivc, inferior vena cava.

(Reproduced with permission from Ref. [109].)

albumin nanoparticles for use as induction chemotherapy before definitive treatment of advanced squamous cell carcinoma of the tongue was also evaluated on 23 untreated patients who had carcinoma of the tongue. Each patient received two to four infusions, with a three-week interval between infusions. Sixteen patients underwent surgery, and of the remaining seven patients one received chemotherapy alone, four received radiotherapy alone, one received chemotherapy plus radio- 
therapy and one refused any further treatment. Eighteen patients (78\%) had a clinical and radiologic objective response (complete, 26\%; partial, 52\%). Three patients $(13 \%)$ showed stable disease and two (9\%) showed disease progression. The toxicities encountered were hematologic in two patients $(8.6 \%)$ and neurologic in two patients (8.6\%) [110].

Furthermore, a recent phase III trial in patients with metastatic breast cancer compared ABI-007 with paclitaxel. ABI-007 resulted in significantly higher response rates and time to tumor progression than paclitaxel. Toxicity data showed that ABI-007 resulted in less grade 4 neutropenia than paclitaxel, and although the incidence of grade 3 sensory neuropathy was higher with ABI-007, the time until the neuropathy decreased to grade 2 was significantly less with ABI-007 compared with paclitaxel [111].

A cholesterol-rich microemulsion or nanoparticle termed LDE has been developed and its cytotoxicity, pharmacokinetics, toxicity to animals and therapeutic action has been compared with those of the commercial paclitaxel. The cytostatic activity of the drug in the complex was diminished compared with commercial paclitaxel due to the cytotoxicity of the vehicle Cremophor EL used in the commercial formulation. Competition experiments in neoplastic cultured cells showed that paclitaxel oleate and LDE were internalized together by the LDL receptor pathway. LDE-paclitaxel oleate arrested the $\mathrm{G}_{2} / \mathrm{M}$ phase of cell cycle, similarly to commercial paclitaxel. Tolerability to mice was remarkable, such that the lethal dose $\left(\mathrm{LD}_{50}\right)$ was nine-fold greater than that of the commercial formulation. Furthermore, LDE concentrated paclitaxel oleate in the tumor four-fold relative to the normal adjacent tissues. At equimolar doses, the association of paclitaxel oleate with LDE resulted in remarkable changes in the drug pharmacokinetic parameters when compared to commercial paclitaxel, with increasing half-life, area under the time-concentration curve and diminishing clearance [112].

The in vitro antitumoral activity of a developed poly(lactic-co-glycolic acid) nanoparticle formulation incorporating paclitaxel-loaded has been assessed on a human small cell lung cancer cell line (NCI-H69 SCLC) and compared to the in vitro antitumoral activity of the commercial formulation. The release behavior of paclitaxel from the nanoparticles exhibited a biphasic pattern characterized by an initial fast release during the first $24 \mathrm{~h}$, followed by a slower and continuous release. Incorporation of paclitaxel in these nanoparticles strongly enhanced the cytotoxic effect of the drug as compared to free drug, this effect being more relevant for prolonged incubation times [113]. In addition, nanoparticles of poly(lactic-co-glycolic acid) have been developed by a modified solvent extraction/evaporation technique, in which natural emulsifiers, such as phospholipids, cholesterol and vitamin E TPGS were applied. These natural emulsifiers showed great advantages for nanoparticle formulation of paclitaxel over the traditional macromolecular emulsifiers such as poly(vinyl alcohol). In the human adenocarcinoma cell line HT-29 the cytotoxicity caused by the drug administered after $24 \mathrm{~h}$ incubation was 13-fold higher than that caused by the free drug [114].

To improve paclitaxel use in intravesical therapy of superficial bladder cancer, paclitaxel-loaded gelatine nanoparticles have been developed. The excipient Cremo- 
phor EL contained in the commercial formulation of paclitaxel forms micelles that entrap the drug, reducing its partition across the urothelium. The paclitaxel-loaded nanoparticles were active against human RT4 bladder transitional cancer cells with $\mathrm{IC}_{50} \mathrm{~S}$ nearly identical to those of the commercial solution of paclitaxel. In dogs given an intravesical dose of paclitaxel-loaded particles, drug concentrations in the urothelium and lamina propria tissue layers, where Ta and T1 tumors would be located, were 2.6-fold greater than those reported for dogs treated with the Cremophor EL formulation [115].

The in vitro cytotoxicity, in vivo antitumor activity, pharmacokinetics, pharmacodynamics, and neurotoxicity of a micellar nanoparticle formulation of paclitaxel, NK105, has been compared with those of free paclitaxel. NK105 showed significantly potent antitumor activity on a human colorectal cancer cell line (HT-29) xenograft as compared with paclitaxel. The area under the time-concentration curve was approximately 90 -fold higher for NK105 than for free paclitaxel. Leakage of paclitaxel from normal blood vessels was minimal and its capture by the reticuloendothelial system minimized. Thus, the tumor area under the time-concentration curve was 25-fold higher for NK105 than for free paclitaxel. Neurotoxicity was significantly weaker with NK105 than with free paclitaxel [116].

Paclitaxel-loaded biodegradable nanoparticles following conjugation to transferrin ligand have been developed to enhance the therapeutic efficacy of the encapsulated drug in the treatment of prostate cancer. The antiproliferative activity of nanoparticles was determined in a human prostate cancer cell line (PC3) and the effect on tumor inhibition in a murine model of prostate cancer. The $\mathrm{IC}_{50}$ of the drug with transferrin ligand conjugated nanoparticles was about five-fold lower than that with unconjugated nanoparticles or drug in solution. Animals that received an intratumoral injection of paclitaxel loaded transferrin ligand conjugated nanoparticles demonstrated complete tumor regression and greater survival rate than those that received either unconjugated nanoparticles or paclitaxel Cremophor EL formulation [117].

Poly(ethylene glycol)-coated biodegradable poly(cyanoacrylate) nanoparticles were also conjugated to transferrin for paclitaxel delivery. This nanoparticle formulation exhibited a markedly delayed blood clearance in mice and the paclitaxel level remained much higher at $24 \mathrm{~h}$ compared with that of free drug after paclitaxel intravenous injection. In S-180 solid tumor-bearing mice, tumor regression was significant with the actively targetable nanoparticles, and complete tumor regression occurred for five out of nine mice. In addition, the life span of tumor-bearing mice was significantly increased when they were treated with the nanoparticle formulation [118].

Paclitaxel is active against gliomas and various brain metastases, though its use in treating brain tumors is limited due to low blood-brain barrier permeability. The lack of paclitaxel brain uptake is thought to be associated with the Pglycoprotein efflux transporter. To improve paclitaxel brain uptake, paclitaxel was entrapped in ethyl alcohol/polysorbate nanoparticles. The paclitaxel nanoparticles cytotoxicity profile was monitored in two different cell lines, U-118 and HCT-15. Brain uptake of paclitaxel nanoparticles was evaluated using an in situ rat brain 
perfusion model. The results suggest that entrapment of paclitaxel in nanoparticles significantly increases the drug brain uptake and its toxicity toward P-glycoproteinexpressing tumor cells. It was hypothesized that paclitaxel nanoparticles could mask paclitaxel characteristics and thus limit its binding to P-glycoprotein, which consequently would lead to higher brain and tumor cell uptake of the otherwise effluxed drug [119].

1.4.7

Miscellaneous Agents

\subsubsection{Arsenic Trioxide}

Arsenic trioxide was considered as a novel antitumor agent. However, it also showed a severe toxicity effect on normal tissue. To improve its therapeutic efficacy and decrease its toxicity, arsenic trioxide-loaded albuminutes immuno-nanospheres targeted with monoclonal antibody (McAb) BDI-1 have been developed and its specific killing effect against bladder cancer cells (BIU-87) investigated. The albuminutes immuno-nanospheres were tightly junctioned with the BIU-87 cells and specific killing activity of bladder tumor cells was observed [120].

\subsubsection{Butyric Acid}

Butyric acid, a short-chain fatty acid naturally present in the human colon, regulates cell proliferation. It specifically modulates the expression of oncogenes such as c-myc, c-fos and $\mathrm{H}$-ras, and various genes involved in the activation of apoptosis like p53 and bcl-2. The clinical applicability of the sodium salt of butyric acid is limited because of its short half-life of approximately $5 \mathrm{~min}$. To improve its efficacy the pro-drug cholesterylbutyrate has been used as a lipid matrix to develop SLNs. The in vitro antiproliferative effect of cholesterylbutyrate SLNs was stronger than that of sodium butyrate in several human cell lines [121-123].

\subsubsection{Cystatins}

Cystatins can inhibit the tumor-associated activity of intracellular cysteine proteases cathepsins B and L and have been suggested as potential anticancer drugs. Chicken cystatin, a model protein inhibitor of cysteine proteases, in poly(lactide-coglycolide) nanoparticles has been developed to improve its bioavailability and delivery into tumor cells. Poly(lactide-co-glycolide) nanoparticles and cystatin-loaded poly(lactic-co-glycolic acid) (PLGA) nanoparticles were cytotoxic towards mammary MCF-10A neoT cells, but free cystatin at the same concentrations was not. Poly (lactide-co-glycolide) nanoparticles were rapidly internalized into MCF-10A neoT cells, whereas the uptake of free cystatin was very slow. These nanoparticles are a useful carrier system. Delivery of the protein inhibitor into tumor cells was rapid and useful to inhibit intracellular proteolysis [124].

\subsubsection{Diethylenetriaminepentaacetic Acid}

Since chelating agents exhibit anticancer effects, the cytotoxicity of the extracellular chelator diethylenetriaminepentaacetic acid (DTPA) has been evaluated in breast 
cancer cells, MCF-7, and neuroblastoma cells, UKF-NB-3. DTPA inhibited cancer cell growth in three-fold lower concentrations compared to human foreskin fibroblasts. The anticancer activity of chelating agents is caused by intracellular complexation of metal ions. DTPA was covalently coupled to human serum albumin nanoparticles and gelatin type B (GelB) nanoparticles to increase its cellular uptake. Coupling of DTPA to this drug carrier system increased its cytotoxic activity by five-fold [125].

\subsubsection{Mitoxantrone}

Mitoxantrone-loaded poly(butyl cyanoacrylate) nanoparticles have been tested in leukemia- or melanoma-bearing mice after intravenous injection. Efficacy and toxicity of mitoxantrone nanoparticles were compared with a drug solution and with a mitoxantrone-liposome formulation. The poly(butyl cyanoacrylate) nanoparticles and liposomes influenced the efficacy of mitoxantrone in cancer therapy in different ways. Liposomes prolonged survival time in P388 leukemia, whereas nanoparticles led to a significant tumor volume reduction in B16 melanoma. Neither nanoparticles nor liposomes were able to reduce the toxic side-effects caused by mitoxantrone, specifically leucocytopenia [126].

In a different study, mitoxantrone adsorbed onto poly(isobutyl cyanoacrylate) nanospheres, coated or not with poloxamine 1508, was administered by intravenous injection in B16 melanoma-bearing mice. In both cases, the observed tumor concentrations of mitoxantrone were high. However, the influence of the hydrophilic coating of the nanoparticles on the biodistribution and pharmacokinetics was minor. Moreover, the non-adsorbed drug was not removed from the nanoparticle preparation and the hydrophilic coating was rapidly desorbed in vivo [127].

\subsection{8}

\section{Gene Therapy}

Delivery systems are necessary for molecules such as antisense oligonucleotides since they are susceptible to nuclease-mediated degradation in the circulation and penetrate poorly through the membranes. They are also susceptible to nuclease attack within the lysosomes and their site of action is either in the cytoplasm in the case of an antisense strategy or in the nucleus for gene replacement or antigene therapy.

Antisense oligonucleotides are molecules that can inhibit gene expression being potentially active for the treatment of cancer. Short nucleic acid sequences specific to oncogene targets such as bcl-2, bcr-abl and c-myc have been shown to exhibit specific anticancer activity in vitro through antigene or antisense activity. However, their negative charge seriously hinders the intracellular penetration of these short fragments of nucleic acid. Efficient in vivo delivery of oligonucleotides remains a major limitation for their therapeutic application.

To prevent the degradation of oligonucleotides and improve their intracellular capture, it was proposed to associate them with nanoparticles. Oligonucleotides associated to nanoparticles were shown to be protected against degradation and to 
penetrate more easily into different types of cells. Poly(alkyl cyanoacrylate) nanoparticles mainly release their drug content by biodegradation, thus rendering the release profile of an entrapped compound independent of its physicochemical characteristics. After intratumoral injection, the bioavailability of oligonucleotides is seriously reduced due to their fast degradation by ubiquitous exo- and endonucleases [128].

For instance, a carrier system consisting of a cationic hydrophobic detergent (cetyltrimethylammonium bromide, CTAB), which interacted with the oligonucleotides by ion-pairing, was developed. In vivo, HBL100ras1 cells implanted in nude mice were treated intratumorally with several formulations of oligonucleotides targeted against Has-ras oncogene. Tumor growth inhibition was achieved at concentrations 100-fold lower than those needed with free oligonucleotides, when the oligonucleotides-CTAB complex was adsorbed onto the surface of the poly(isohexyl cyanoacrylate) nanospheres. Interestingly, the oligonucleotides-CTAB complex alone exerted no effect on HBL1000ras1 cell proliferation. Analysis of the amount of intact intracellular oligonucleotides, in cell culture experiments, revealed concentrations 100 -fold higher in cells treated with oligonucleotides-CTAB adsorbed onto nanospheres [129].

The nanospheres were able to enhance oligonucleotides cell internalization and to protect oligonucleotides from both rapid cell internalization and rapid intracellular breakdown, which led to a considerably higher intracellular concentration of intact oligonucleotides and to a more efficient antisense activity. However, the oligonucleotides release was followed by the release of the detergent CTAB, which, at high intracellular concentrations, could induce cell toxicity.

Cholesterol-modified oligonucleotides, capable of direct adsorption onto poly(alkyl cyanoacrylate) nanospheres without the need for potentially toxic intermediates, have also been tested, but they proved less able to inhibit T24 human bladder carcinoma cells proliferation in culture than the system previously described [130].

To circumvent CTAB toxicity while maintaining an efficient biological activity, another approach was developed. Functional nanospheres were obtained by free radical emulsion polymerization of methyl methacrylate using the quaternary ammonium salt of 2-(dimethylamino)ethyl methacrylate as the reactive emulsifier [131].

The cationic dextran derivative diethylaminoethyl-dextran, by formation of an ion pair at the oligonucleotides surface, was developed to replace CTAB and was associated with poly(isohexyl cyanoacrylate) nanospheres. Poly(isohexyl cyanoacrylate) nanoparticles, with an aqueous core containing oligonucleotides, were prepared by interfacial polymerization of isobutyl cyanoacrylate in water/oil emulsion. Further studies also demonstrated that nanoencapsulation was able to protect oligonucleotides against degradation by serum nucleases contained in the cell culture medium [132].

For in vivo studies, phosphorothioate oligonucleotides against EWS Fli-1 chimeric RNA were encapsulated into cyanoacrylate nanocapsules and their efficacy was tested on experimental Ewing sarcoma, after intratumoral administration. Oligo- 
nucleotides nanocapsules led to more efficient tumor growth inhibition. The encapsulation of oligonucleotides provided enhanced protection against in vivo degradation, resulting in a higher number of oligonucleotides available. Cyanoacrylate nanocapsules yielded a higher ratio of oligonucleotides targeting the tumor cells [133].

In vivo, poly(alkyl cyanoacrylate) nanoparticles were able to efficiently distribute the oligonucleotides to the liver and to improve, in mice, the treatment of RAS cells expressing the point mutated Ha-ras gene. As all the oligonucleotides studied had a specific therapeutic efficacy, this means that the oligonucleotides delivered by nanoparticles escape from the lysosome compartment before degradation [134].

Preclinical studies have shown that DOTAP:cholesterol nanoparticles are effective systemic gene delivery vectors that efficiently deliver tumor suppressor genes to disseminated lung tumors. A phase-I trial for systemic treatment of lung cancer using a novel tumor suppressor gene, FUS1, has been initiated. Although DOTAP: cholesterol nanoparticles complexed to DNA are efficient vectors for systemic therapy, induction of an inflammatory response in a dose-dependent manner has also been observed, thus limiting its use. Systemic administration of DNA nanoparticles induced multiple signaling molecules both in vitro and in vivo that are associated with inflammation. Use of small molecule inhibitors against the signaling molecules resulted in their suppression and thereby reduced inflammation without affecting transgene expression [135]. Nanoparticles coated with ligands such as transferrin and epidermal growth factor (EGF) have also been developed in gene delivery to target selectively the tumor cells [136]. Complexes for DNA delivery composed of polyethylenimine (polyplexes) linked to poly(ethylene glycol) and coated with transferrin or EGF were prepared with fixed nanoparticle diameters. Intravenous injection of the transferrin-coated polyplexes resulted in gene transfer to subcutaneous neuroblastoma tumors in syngenic mice, and intravenous injection of the EGF-coated polyplexes targeted human hepatocellular carcinoma xenografts in SCID mice. In these models, luciferase marker gene expression levels in tumor tissues were 10- to 100-fold higher than in other organ tissues. Repeated systemic application of the transferrin polyplexes encoding tumor necrosis factor alpha (TNF- $\alpha$ ) into tumor-bearing mice induced tumor necrosis and inhibition of tumor growth in different murine tumor models [137].

Transferrin-modified nanoparticles containing DNAzymes (short catalytic singlestranded DNA molecules) for tumor targeting have been developed. Linear, $\beta$ cyclodextrin-based polymers were complexed with DNAyzme molecules to form sub-50 nm particles termed "polyplexes". Adamantane forms inclusion complexes with the surface cyclodextrins of the polyplexes, allowing a sterically stabilizing layer of poly(ethylene glycol) to be added. The stabilized polyplexes were also modified with transferrin to increase the targeting to tumor cells expressing transferrin receptors. Administration by intraperitoneal bolus, infusion, intravenous bolus and subcutaneous injection were studied in tumor-bearing nude mice. DNAzymes packaged in polyplex formulations were concentrated and retained in tumor tissue and other organs, whereas unformulated DNAzyme was eliminated from the body 
within $24 \mathrm{~h}$ post-injection. Tumor cell uptake was observed with intravenous bolus injection only and intracellular delivery requires transferrin targeting [138].

Potent sequence selective gene inhibition by siRNA is also hindered by poor intracellular uptake, limited blood stability and non-specific immune stimulation. Thus, ligand-targeted, sterically stabilized nanoparticles have been developed for siRNA. Self-assembling nanoparticles with siRNA were constructed with poly(ethyleneimine) that is PEGylated with an Arg-Gly-Asp peptide ligand, as a means to target tumor neovasculature expressing integrins, and used to deliver siRNA inhibiting vascular endothelial growth factor receptor-2 (VEGF R2) expression and thereby tumor angiogenesis. Intravenous administration into tumor-bearing mice gave selective tumor uptake, siRNA sequence-specific inhibition of protein expression within the tumor and inhibition of both tumor angiogenesis and growth rate [139].

\section{References}

1 I. Brigger, C. Dubernet, P. Couvreur, Nanoparticles in cancer therapy and diagnosis, Adv. Drug Deliv. Rev. 2002, 54, 631-651.

2 R.K.Y. Zee-Cheng, C.C. Cheng, Delivery of anticancer agents, Meth. Find. Exp. Clin. Pharmacol. 1989, 11, 439-529.

3 G. Barratt, Colloidal drug carriers: Achievements and perspectives, Cell. Mol. Life Sci. 2003, 60, 21-37.

4 R.K. JaIn, Transport of molecules in the tumor interstitium: A review, Cancer Res. 1987, 47, 3039-3051.

5 D. Baban, L.W. Seymour, Control of tumor vascular permeability, Adv. Drug Deliv. Rev. 1998, 34, 109-119.

6 S.K. Новbs, W.L. Monsky, F. Yuan, W.G. Roberts, L. Griffith, V.P. Torchilin, R.K. JaIn, Regulation of transport pathway in tumor vessels: Role of tumor type and microenvironment, Proc. Natl. Acad. Sci. U.S.A. 1998, 95, 4607-4612.

7 H. MAEDA, The enhanced permeability and retention (EPR) effect in tumor vasculature: The key role of tumorselective macromolecular drug targeting, Adv. Enzyme Regul. 2001, 41, 189-207.

8 R. Krishna, L.D. Mayer, Multidrug resistance (MDR) in cancer- mechanisms, reversal using modulators of MDR and the role of MDR modulators in influencing the pharmacokinetics of anticancer drugs, Eur. J. Cancer Sci. 2000, 11, 265-283.

9 A.K. Larsen, A.E. Escargueil, A. SkLADANowski, Resistance mechanisms associated with altered intracellular distribution of anticancer agents, Pharmacol. Ther. 2000, 88, 217-229.

10 C. Vauthier, C. Dubernet, C. Chauvierre, I. Brigger, P. Couvreur, Drug delivery to resistant tumors: The potential of poly(alkyl cyanoacrylate) nanoparticles, $J$. Control. Release 2003, 93, 151-160.

11 J. Sinek, H. Frieboes, X. Zheng, V. Cristini, Two-dimensional chemotherapy simulations demonstrate fundamental transport and tumor response limitations involving nanoparticles, Biomed. Microdevices 2004, 6, 297-309.

12 V.S. Shenoy, I.K. ViJay, R.S. Murthy, Tumour targeting: Biological factors and formulation advances in injectable lipid nanoparticles, $J$. Pharm. Pharmacol. 2005, 57, 411422.

13 B. Rinova, Immunomodulating activities of soluble synthetic polymer- 
bound drugs, Adv. Drug Deliv. Rev. 2002, 54, 653-674.

14 G. Storm, S.O. Belliot, T. Daemen, D.D. LASIC, Surface modification of nanoparticles to oppose uptake by the mononuclear phagocyte system, Adv. Drug Deliv. Rev. 1995, 17, 31-48.

15 G.M. Barrat, F. Puisieux, W.P. Yu, C. Foucher, H. Fessi, J.P. Devissaguet, Anti-metastatic activity of MDP-L-alanyl-cholesterol incorporated into various types of nanocapsules, Int. J. Immunopharm. 1994, 457-461.

16 P. Couvreur, B. Kante, L. Grislain, M. Roland, P. Speiser, Toxicity of polyalkykyanoacrylate nanoparticles II: Doxorubicin-loaded nanoparticles, J. Pharm. Sci. 1982, 71, 790-792.

17 P.M. Blagoeva, R.M. Balansky, T.J. Mircheva, M.I. Simeonova, Diminished genotoxicity of mitomycin $\mathrm{C}$ and farmorubicin included in polybutylcyanoacrylate nanoparticles, Mut. Res. 1992, 268, 77-82.

18 C. Verdun, F. Brasseur, H. Vranckx, P. Couvreur, M. Roland, Tissue distribution of doxorubicin associated with polyisohexylcyanoacrylate nanoparticles, Cancer Chemother. Pharmacol. 1990, 26, 13-18.

19 R. Fernandez-Urrusuno, E. Fattal, J.M. Rodrigues, J. Féger, P. Bedossa, P. Couvreur, Effect of polymeric nanoparticle administration on the clearance activity of the mononuclear phagocyte system in mice, J. Biomed. Mater. Res. 1996, 31, 401-408.

20 F. Fawaz, F. Bonini, M. Guyot, A.M. Lagueny, H. Fessi, J.P. Devissaguet, Influence of poly(DL-lactide) nanocapsules on the biliary clearance and enterohepatic circulation of indomethacin in the rabbit, Pharm. Res. 1993, 10, 750-766.

21 S. Gibaud, J.P. Andreux, C. Weingarten, M. Renard, P. Couvreur, Increased bone marrow toxicity of doxorubicin bound to nanoparticles, Eur. J. Cancer 1994, 30A, 820-826.

22 S.M. Moghimi, A.C. Hunter, J.C. Murray, Long-circulating and target- specific nanoparticles: Theory to practice, Pharmacol. Rev. 2001, 53, 283-318.

23 S. Gibaud, C. Rousseau, C. Weingarten, R. Favier, L. Douay, J.P. Andreux, P. Couvreur, Polyalkylcyanoacrylate nanoparticles as carriers for granulocyte colony stimulating factor (G-CSF), J. Control. Release 1998, 52, 131-139.

24 L. Illum, L.O. Jacobsen, R.H. MÜller, R. MAK, S.S. Davis, Surface characteristics and the interaction of colloidal particles with mouse peritoneal macrophages, Biomaterials 1987, 8, 113-117.

25 S.I. Jeon, J.H. Lee, J.D. Andrade, P.G. De Gennes, Protein-surface interactions in the presence of polyethylene oxide: Simplified theory, J. Colloid. Interface Sci. 1991, 142, 149-158.

26 R. Gref, A. Domb, P. Quellec, T. BlunK, R.H. MÜlLER, J.M. Verbavatz, R. LANGer, The controlled intravenous delivery of drugs using PEG-coated sterically stabilized nanospheres, Adv. Drug Deliv. Rev. 1995, 16, 215-233.

27 D. Bazile, C. Prud'Homme, M.T. Bassoultet, M. Marlard, G. Spenlehauer, M. Veillard, Stealth Me.PEG-PLA nanoparticles avoid uptake by the mononuclear phagocyte system, J. Pharm. Sci. 1995, 84, 493498.

28 V.C.F. Mosqueira, P. Legrand, A. Gulik, O. Bourdon, R. Gref, D. Labarre, G. Barratt, Relationship between complement activation, cellular uptake and surface physicochemical aspects of novel PEGmodified nanocapsules, Biomaterials 2001, 22, 2967-2979.

29 V. Lenaerts, A. Labib, F. Choinard, J. Rousseau, H. Ali, J. van Lier, Nanocapsules with a reduced liver uptake: Targeting of phthalocyanines to EMT-6 mouse mammary tumor in vivo, Eur. J. Pharm. Biopharm. 1995, $41,38-43$.

30 O. Bourdon, V. Mosqueira, P. LEGRAND, J. Blais, A comparative study of the cellular uptake, 
localization and phototoxicity of metatetra(hydroxyphenyl) chlorin encapsulated in surface-modified submicronic oil/water carriers in HT29 tumor cells, J. Photochem. Photobiol. B 2000, 55, 164-171.

31 M.T. Peracchia, C. Vauthier, F. Puisieux, P. Couvreur, Development of sterically stabilized poly(isobutyl 2-cyanoacrylate) nanoparticles by chemical coupling of poly(ethylene glycol), J. Biomed. Mater. Res. 1997, 34, 317-326.

32 I. Brigger, P. Chaminade, V. Marsaud, M. Appel, M. Besnard, R. Gurny, M. Renoir, P. Couvreur, Tamoxifen encapsulation within polyethylen glycol-coated nanospheres: A new antiestrogen formulation, Int. J. Pharm. 2001, 214, 37-42.

33 S.E. Ghelperina, Z.S. Smirnova, A.S. Khalanskiy, I.N. Skidan, A.I. Bobruskin, J. Kreuter, Chemotherapy of brain tumours using doxorubicin bound to polysorbate 80coated nanoparticles, Proceedings of $3^{\text {rd }}$ World Meeting APV/APGI, Berlin, Germany 2000, 441-442.

$34 \mathrm{~J}$. KReUter, Influence of the surface properties on nanoparticle-mediated transport of drugs to the brain, J. Nanosci. Nanotechnol. 2004, 4, 484-488.

35 E. Allémann, J. Rousseau, N. Brasseur, S.V. Kudrevich, K. Lewis, J.E. van Lier, Photodynamic therapy of tumours with hexadecafluoro zinc phthalocyanine formulated in PEGcoated poly(lactic acid) nanoparticles, Int. J. Cancer 1996, 66, 821-824.

36 J. Lode, I. Fichtner, J. Kreuter, A. Berndt, J.E. Diederichs, R. ReszKa, Influence of surface-modifying surfactants on the pharmacokinetic behaviour of 14C-poly(methylmethacrylate) nanoparticles in experimental tumor models, Pharm. Res. 2001, 18, 16131619.

37 M.T. Peracchia, C. Vauthier, D. Desmaële, A. Gulik, J.C. Dedieu, M. Demoy, J. D'Angelo, P. Couvreur, Pegylated nanoparticles from a novel methoxypolyethylene glycol cyanoacrylate-hexadecyl cyanoacrylate amphiphilic copolymer, Pharm. Res. 1998, 15, 550-556.

38 S.E. Dunn, A.G.A. Coоmbes, M.C. Garnett, S.S. Davis, M.C. Davies, L. ILLUM, In vitro interaction and in vivo biodistribution of poly(lactideglycolide) nanospheres surface modified by poloxamer and poloxamine copolymers, J. Control. Release 1997, 44, 65-76.

39 S. Stolnik, S.E. Dunn, M.C. Garnett, M.C. Davies, A.G.A. Coombes, D.C. TAYlor, M.P. IRving, S.C. Purkiss, T.F. Tadros, S.S. Davis, Surface modification of poly(lactideco-glycolide) nanospheres by biodegradable poly(lactide)poly(ethylene glycol) copolymers, Pharm. Res. 1994, 11, 1800-1808.

40 V.C.F. Mosqueira, P. Legrand, R. Gref, B. Heurtault, M. Appel, G. BARRATT, Interactions between a macrophage cell line (J774A1) and surface-modified poly(D,L-lactide) nanocapsules bearing poly(ethylene glycol), J. Drug Target. 1999, 7, 6578.

41 V.C.F. Mosqueira, P. Legrand, A. Gulik, O. Bourdon, R. Gref, D. LABARre, G. BARRATt, Biodistribution of novel long circulating PEG-grafted nanocapsules in mice: Effects of PEG chain length and density, Pharm. Res. 2001, 18, 1411-1419.

42 B. Stella, S. Arpicco, M.T. Peracchia, D. Desmaële, J. Hoebene, M. Renoir, J. D'Angelo, L. Cattel, P. Couvreur, Design of folic acidconjugated nanoparticles for drug targeting, J. Pharm. Sci. 2000, 89, 1452-1464.

43 L. Zhang, S. Hou, S. Mao, D. Wei, X. Song, Y. Lu, L. Zhang, S. Hou, S. MaO, D. Wei, X. Song, Y. Lu, Uptake of folate-conjugated albumin nanoparticles to the SKOV3 cells, Int. J. Pharm. 2004, 287, 155-162.

44 T. Shiokawa, Y. Hattori, K. Kawano, Y. Ohguchi, H. Kawakami, K. Toma, Y. Maitani, Effect of polyethylene glycol linker chain length of folate-linked microemulsions loading aclacinomycin A on targeting ability and antitumor effect in vitro 
and in vivo, Clin. Cancer Res. 2005, 11, 2018-2025.

45 N.C. Belloce, S.H. Pun, G.S. Jensen, M.E. DAvis, Transferrin-containing, cyclodextrin polymer-based particles for tumor-targeted gene delivery, Bioconj. Chem. 2003, 14, 1122-1132.

46 H. Wartlick, K. Michaelis, S. Balthasar, K. STREbhardT, J. Kreuter, K. Langer, Highly specific HER2-mediated cellular uptake of antibody-modified nanoparticles in tumour cells, J. Drug Target. 2004, 12, 461-471.

47 E.M. Gordon, F.L. Hall, Nanotechnology blooms, at last, Oncol. Rep. 2005, 13, 1003-1007.

48 F.B. Landry, D.V. Bazile, G. Spenlehauer, M. Veillard, J. Kreuter, Peroral administration of 14C-poly-(D,L-lactic acid) nanoparticles coated with human serum albumin or polyvinyl alcohol to guinea pigs, J. Drug Target 1998, 6, 293-307.

49 G. Ponchel, J.M. Irache, Specific and non-specific bioadesive particulate systems for oral delivery to the gastrointestinal tract, Adv. Drug Deliv. Rev. 1998, 34, 191-219.

50 L. Mu, S.S. FenG, A novel controlled release formulation for the anticancer drug paclitaxel (Taxol): PLGA nanoparticles containing vitamin $\mathrm{E}$ TPGS, J. Control. Release 2003, 86, 33-48.

51 Y. Nishioka, H. Yoshino, Lymphatic targeting with nanoparticulate system, Adv. Drug Deliv. Rev. 2001, 47, 55-64.

52 C. Sakakura, T. Takahashi, K. Sawai, A. Hagiwara, M. Ito, S. Shobayashi, S. Sasaki, K. Ozaki, M. Shirasu, Enhancement of therapeutic efficacy of aclarubicin against lymph node metastases using a new dosage form: Aclarubicin adsorbed on activated carbon particles, Anti-Cancer Drugs 1992, 3, 233-236.

53 A. Hagiwara, T. Takahashi, K. Sawai, C. Sakakura, M. Shirasu, M. OHgaki, T. Imanashi, J. Yamasaki, Y. Takemoto, N. Kageyama, Selective drug delivery to peri-tumoral region and regional lymphatics by local injection of aclarubicin adsorbed on activated carbon particles in patients with breast cancer - a pilot study. AntiCancer Drugs 1997, 8, 666-670.

54 A.E. Hawley, S.S. Davis, L. Illum, Targeting of colloids to lymph nodes: Influence of lymphatic physiology and colloidal characteristics, Adv. Drug Deliv. Rev. 1995, 17, 129-148.

55 H. Tokumitsu, J. Hiratsuka, Y. SKurai, T. Kobayashi, H. ICHIKaWA, Y. FUKUMORI, Gadolinium neutroncapture therapy using novel gadopentetic acid-chitosan complex nanoparticles: In vivo growth suppression of experimental melanoma solid tumor, Cancer Lett. 2000, 150, 177-182.

56 P. Calvo, J.L. Vila-Jato, M.J. Alonso, Evaluation of cationic polymer-coated nanocapsules as ocular drug carriers, Int. J. Pharm. 1997, 153, 41-50.

57 G. Minotti, P. Menna, E. Salvatorelli, G. Cairo, L. Gianni, Anthracyclines: Molecular advances and pharmacological developments in antitumor activity and cardiotoxicity. Pharmacol. Rev. 2004, 56, 185-229.

58 N. Chiannilkulchai, Z. Driouich, J.P. Benoit, A.L. Parodi, P. Couvreur, Doxorubicin-loaded nanoparticles: Increased efficiency in murine hepatic metastasis, Sel. Cancer Ther. 1989, 5, 1-11.

59 N. Chiannilkulchai, N. Ammoury, B. Caillou, J.Ph. Devissaguet, P. Couvreur, Hepatic tissue distribution of doxorubicin-loaded particles after i.v. administration in reticulosarcoma M 5076 metastasis-bearing mice, Cancer Chemother. Pharmacol. 1990, 26, 122-126.

60 A. Rolland, Clinical pharmacokinetics of doxorubicin in hepatoma patients after a single intravenous injection of free or nanoparticle-bound anthracycline, Int. J. Pharm. 1989, 54, 113-121.

61 J. Kattan, J.P. Droz, P. Couvreur, J.P. Marino, A. Boutan-Laroze, P. Rougier, P. Brault, H. Vranckx, J.M. Grognet, X. Morge, H. Sancho-Garnier, Phase I clinical trial and pharmacokinetics evaluation of doxorubicin carried by poly- 
isohexylcyanoacrylate nanoparticles, Invest. New Drugs 1992, 10, 191-199.

62 C.E. Soma, C. Dubernet, G. Barratt, S. Benita, P. Couvreur, Investigation of the role of macrophages on the cytotoxicity of doxorubicin and doxorubicin-loaded nanoparticles on M5076 cells in vitro, J. Control. Release 2000, 68, 283-289.

63 S. Mitra, U. Gaur, P.C. Gosh, A.N. MaItRA, Tumor targeted delivery of encapsulated dextran-doxorubicin conjugate using chitosan nanoparticles as carrier, J. Control. Release 2001, 74, 317-323.

64 J.H. Chen, R. Ling, Q. YaO, L. Wang, Z. MA, Y. LI, Z. WANG, H. Xu, Enhanced antitumor efficacy on hepatoma-bearing rats with adriamycin-loaded nanoparticles administered into hepatic artery. World J. Gastroenterol. 2004, 10(13), 19891991.

65 J.H. Chen, L. Wang, R. Ling, Y. Li, Z. WANG, Q. YAO, Z. MA, Body distribution of nanoparticle-containing adriamycin injected into the hepatic artery of hepatoma-bearing rats, Dig. Dis. Sci. 2004, 49, 1170-1173.

66 L. Manil, P. Couvreur, P. Mahieu, Acute renal toxicity of doxorubicin (adryamycin)-loaded cyanoacrylate nanoparticles, Pharm. Res. 1995, 12 , 85-87.

67 E. Leo, R. Arletti, F. Forni, R. Cameroni, General and cardiac toxicity of doxorubicin-loaded gelatin nanoparticles, Il Farmaco 1997, 52, 385-388.

68 J. Kreuter, Nanoparticulate systems for brain delivery of drugs, Adv. Drug Deliv. Res. 2000, 47, 65-81.

69 S.E. Gelperina, A.S. Khalansky, I.N. Skidan, Z.S. Smirnova, A.I. Bobruskin, S.E. Severin, B. Turowski, F.E. Zanella, J. Kreuter, Toxicological studies of doxorubicin bound to polysorbate 80-coated poly(butyl cyanoacrylate) nanoparticles in healthy rats and rats with intracranial glioblastoma, Toxicol. Lett. 2002, 126, 131-141.

70 S.C. Steiniger, J. Kreuter, A.S. Khalansky, I.N. Skidan, A.I.
Bobruskin, Z.S. SMirnova, S.E. SeVterin, R. Uhl, M. Kock, K.D. Geiger, S.E. Gelperina, Chemotherapy of glioblastoma in rats using doxorubicin-loaded nanoparticles, Int. J. Cancer 2004, 109, 759-767.

71 H.S. Yoo, K.H. LeE, J.E. OH, T.G. PARK, In vitro and in vivo anti-tumor activities of nanoparticles based on doxorubicin-PLGA conjugates, J. Control. Release 2000, 68, 419-431.

72 L.H. Reddy, R.K. Sharma, R.S. MuRTHY, Enhanced tumour uptake of doxorubicin loaded poly(butyl cyanoacrylate) nanoparticles in mice bearing Dalton's lymphoma tumour, J. Drug Target 2004, 12, 443-451.

73 I. Brigger, J. Morizet, L. Laudani, G. Aubert, M. Appel, V. Velasco, M.J. Terrier-Lacombe, D. Desmaele, J. D'Angelo, P. Couvreur, G. Vassal, Negative preclinical results with stealth nanospheres-encapsulated doxorubicin in an orthotopic murine brain tumor model, J. Control. Release 2004, 100, 29-40.

74 R.H. Müller, K. Mäder, S. Gohla, Solid lipid nanoparticles (SLN) for controlled drug delivery - a review of the state of the art, Eur. J. Pharm. Biopharm. 2000, 50, 161-177.

75 R.N. Alyautdin, V.E. Petrov, K. LANGer, A. Berthold, D.A. Kharkevich, J. Kreuter, Delivery of loperamide across the blood-brain barrier with Polysorbate 80-coated polybutylcyanoacrylate nanoparticles, Pharm. Res. 1997, 14, 325-328.

76 G.P. Zara, R. Cavalli, A. Fundarò, A. Bargoni, O. Caputo, M.R. Gasco, Pharmacokinetics of doxorubicin incorporated in solid lipid nanospheres (SLN), Pharm. Res. 1999, 44, 281-286.

77 A. Miglietta, R. Cavalli, C. Bocca, L. Gabriel, M.R. Gasco, Cellular uptake and cytotoxicity of solid lipid nanospheres (SLN) incorporating doxorubicin or paclitaxel, Int. J. Pharm. 2000, 210, 61-67.

78 L. Serpe, M.G. Catalano, R. Cavalli, E. Ugazio, O. Bosco, R. Canaparo, E. Muntoni, R. Frairia, M.R. Gasco, M. EANDI, G.P. Zara, Cytotoxicity of 
anticancer drugs incorporated in solid lipid nanoparticles on HT-29 colorectal cancer cell line, Eur. J. Pharm.

Biopharm. 2004, 58, 673-680.

79 G.P. Zara, R. Cavalli, A. Bargoni, A. Fundarò, D. Vighetto, M.R. Gasco, Intravenous administration to rabbits of non stealth and stealth doxorubicinloaded solid lipid nanoparticles at increasing concentrations of stealth agent: Pharmacokinetics and distribution of doxorubicin in brain and other tissues, J. Drug Target 2002, 10, 327-335.

80 A. Fundarò, R. Cavalli, A. Bargoni, D. Vighetto, G.P. Zara, M.R. Gasco, Non-stealth and stealth solid lipid nanoparticles (SLN) carrying doxorubicin: Pharmacokinetics and tissue distribution after i.v. administration to rats, Pharm. Res. 2000, 42, 337-343.

81 G.P. Zara, A. Bargoni, R. Cavalli, A. Fundarò, D. Vighetto, M.R. Gasco, Pharmacokinetics and tissue distribution of idarubicin-loaded solid lipid nanoparticles after duodenal administration to rats, J. Pharm. Sci. 2002, 91, 1324-1333.

82 A.C. de Verdière, C. Dubernet, F. Némati, E. Soma, M. Appel, J. Ferté, S. Bernard, F. Puisieux, P. Couvreur, Reversion of multidrug resistance with polyalkylcyanoacrylate nanoparticles: Towards a mechanism of action, Br. J. Cancer 1997, 76, 198 205.

83 A. Astier, B. Doat, M.J. Ferrer, G. Benoit, J. Fleury, A. Rolland, R. LEVERGE, Enhancement of adriamycin antitumor activity by its binding with an intracellular sustained-release form, polymethacrylate nanospheres, in U-937 cells, Cancer Res. 1988, 48, 1835-1841.

84 S. Bennis, C. Chapey, P. Couvreur, J. RoBERT, Enhanced cytotoxicity of doxorubicin encapsulated in polyisohexylcyanoacrylate nanospheres against multi-drug-resistant tumour cells in culture, Eur. J. Cancer 1994, 30A, 889-893.

85 A.C. de Verdière, C. Dubernet, F. Némati, M.F. Poupon, F. Puisieux,
P. Couvreur, Uptake of doxorubicin from loaded nanoparticles in multidrug-resistant leukemic murine cells, Cancer Chemother. Pharmacol. 1994, 33, 504-508.

86 X. Pépin, L. Attali, C. Domraullt, S. Gallet, J.M. Metreau, Y. Reault, P.J. Cardot, M. Imalalen, C. Dubernet, E. Soma, P. Couvreur, On the use of ion-pair chromatography to elucidate doxorubicin release mechanism from polyalkylcyanoacrylate nanoparticles at the cellular level, J. Chromatogr. B 1997, 702, 181-187.

87 J. Nafziger, G. Averland, E. Bertounesque, G. Gaudel, C. Monneret, Synthesis and antiproliferative effects of a $4^{\prime}$ morpholino-9-methyl anthracycline, J. Antibiot. 1995, 48, 1185-1187.

88 C.E. Soma, C. Dubernet, G. Barratt, F. Nemati, M. Appel, S. Benita, P. Couvreur, Ability of doxorubicinloaded nanoparticles to overcome multidrug resistance of tumor cells after their capture by macrophages, Pharm. Res. 1999, 16, 1710-1716.

89 A. Laurand, A. Laroche-Clary, A. Larrue, S. Huet, E. Soma, J. Bonnet, J. ROBERT, Quantification of the expression of multidrug resistancerelated genes in human tumour cell lines grown with free doxorubicin or doxorubicin encapsulated in polyisohexylcyanoacrylate nanospheres, Anticancer Res. 2004, 24, 3781-3788.

90 C.E. Soma, C. Dubernet, D. Bentolila, S. Benita, P. Couvreur, Reversion of multidrug resistance by co-encapsulation of doxorubicin and cyclosporin A in polyalkylcyanoacrylate nanoparticles, Biomaterials 2000, 21, 1-7.

91 T. Ameller, V. Marsaud, P. LEGRAND, R. GREF, J.M. ReNoIr, In vitro and in vivo biologic evaluation of long-circulating biodegradable drug carriers loaded with the pure antiestrogen RU 58668, Int. J. Cancer 2003, 106, 446-454.

92 S. Maillard, T. Amelier, J. Gauduchon, A. Gougelet, F. Gouilleux, P. Legrand, V. Marsaud, E. Fattal, B. Sola, J.M. Renoir, 
Innovative drug delivery nanosystems improve the anti-tumor activity in vitro and in vivo of anti-estrogens in human breast cancer and multiple myeloma, J. Steroid Biochem. Mol. Biol. 2005, 94, 111-121.

93 J.S. Chawla, M.M. Amiji, Cellular uptake and concentrations of tamoxifen upon administration in poly(epsilon-caprolactone) nanoparticles, AAPS Pharm. Sci. 2003, 5, E3.

94 D.B. Shenoy, M.M. Аmiji, Poly(ethylene oxide)-modified poly(epsilon-caprolactone) nanoparticles for targeted delivery of tamoxifen in breast cancer, Int. J. Pharm. 2005, 293, 261-270.

95 D. Bhadra, S. Bhadra, S. Jain, N.K. JAIN, A PEGylated dendritic nanoparticulate carrier of fluorouracil, Int. J. Pharm. 2003, 257, 111-124.

96 C.L. Lo, K.M. Lin, G.H. Hsiue, Preparation and characterization of intelligent core-shell nanoparticles based on poly(D,L-lactide)-g-poly(Nisopropyl acrylamide-co-methacrylic acid), J. Control. Release 2005, 104, 477-488.

97 J.F. Ku Kowska-Latallo, K.A. Candido, Z. Cao, S.S. Nigavekar, I.J. Majoros, T.P. Thomas, L.P. Balogh, M.K. Khan, J.R. JR BaKeR, Nanoparticle targeting of anticancer drug improves therapeutic response in animal model of human epithelial cancer, Cancer Res. 2005, 65, 53175324.

98 S. YANG, J. Zhu, B. Lu, B. Liang, C. YANG, Body distribution of camptothecin solid lipid nanoparticles after oral administration, Pharm. Res. 1999, 16, 751-757.

99 H. Miura, H. Onishi, M. Sasatsu, Y. MACHIDA, Antitumor characteristics of methoxypolyethylene glycol-poly(DLlactic acid) nanoparticles containing camptothecin, J. Control. Release 2004, 97, 101-113.

100 H. Onishi, Y. Machida, Y. Machida, Antitumor properties of irinotecancontaining nanoparticles prepared using poly(DL-lactic acid) and poly(ethylene glycol)-block- poly(propylene glycol)-blockpoly(ethylene glycol), Biol. Pharm. Bull. 2003, 26, 116-119.

101 J. Williams, R. LansDown, R. SWeITZER, M. RoMANowski, R. LaBell, R. Ramaswami, E. Unger, Nanoparticle drug delivery system for intravenous delivery of topoisomerase inhibitors, J. Control. Release 2003, 91, 167-172.

102 K. Avgoustakis, A. Beletsi, Z. Panagi, P. Klepetsanis, A.G. KARYDAS, D.S. ITHAKISSIOS, PLGAmPEG nanoparticles of cisplatin: In vitro nanoparticle degradation, in vitro drug release and in vivo drug residence in blood properties, J. Control. Release 2002, 79, 123-135.

103 A. Barroug, L.T. Kuhn, L.C. Gerstenfeld, M.J. Glimcher, Interactions of cisplatin with calcium phosphate nanoparticles: In vitro controlled adsorption and release, J. Orthop. Res. 2004, 22, 703-708.

104 A.K. Singla, A. Garg, D. Aggarwal, Paclitaxel and its formulations, Int. J. Pharm. 2002, 235, 179-192.

105 A.J. ten Tije, J. Verweij, W.J. Loos, A. Sparreboom, Pharmacological effects of formulation vehicles: Implications for cancer chemotherapy, Clin. Pharmacokinet. 2003, 42, 665685.

106 D. Sharma, T.P. Chelvi, J. Kaur, K. Chakravorty, T.K. De, A. Maitra, R. Ralhan, Novel Taxol formulation: Polyvinylpyrrolidone nanoparticleencapsulated Taxol for drug delivery in cancer therapy, Oncol. Res. 1996, 8 , 281-286.

107 R. Cavalli, O. Caputo, M.R. Gasco, Preparation and characterization of solid lipid nanoparticles incorporating paclitaxel, Eur. J. Pharm. Sci. 2000, 10, 305-309.

108 N.K. Ibrahim, N. Desai, S. Legha, P. Soon-Shiong, R.L. Theriault, E. Rivera, B. Esmaeli, S.E. Ring, A. Bedikian, G.N. Hortobagyi, J.A. ELLERhorst, Phase I and pharmacokinetic study of ABI-007, a cremophor-free, protein-stabilized, nanoparticle formulation of paclitaxel, Clin. Cancer Res. 2002, 8, 1038-1044. 
109 B. Damascelli, G. Cantu, F. Mattavelli, P. Tamplenizza, $P$. Bidoli, E. Leo, F. Dosio, A.M. Cerrotta, G. Di Tolla, L.F. Frigerio, F. Garbagnati, R. Lanocita, A. Marchiano, G. Patelli, C. Spreafico, V. Ticha, V. Vespro, F. Zunino, Intraarterial chemotherapy with polyoxyethylated castor oil free paclitaxel, incorporated in albumin nanoparticles (ABI-007): Phase II study of patients with squamous cell carcinoma of the head and neck and anal canal: Preliminary evidence of clinical activity, Cancer 2001, 92, 25922602.

110 B. Damascelli, G.L. Patelli, R. Lanocita, G. Di Tolla, L.F. Frigerio, A. Marchiano, F. Garbagnati, C. Spreafico, V. Ticha, C.R. Gladin, M. Palazzi, F. Crippa, C. Oldini, S. Calo, A. Bonaccorsi, F. Mattavelit, L. Costa, L. Mariani, G. CANTU, A novel intraarterial chemotherapy using paclitaxel in albumin nanoparticles to treat advanced squamous cell carcinoma of the tongue: Preliminary findings, Am. J. Roentgenol. 2003, 181, 253-260.

111 W.J. Gradishar, The future of breast cancer: The role of prognostic factors, Breast Cancer Res. Treat. 2005, 89, 1726.

112 D.G. Rodrigues, D.A. Maria, D.C. Fernandes, C.J. Valduga, R.D. Couto, O.C. IBANEZ, R.C. MARANHAO, Improvement of paclitaxel therapeutic index by derivatization and association to a cholesterol-rich microemulsion: in vitro and in vivo studies, Cancer Chemother. Pharmacol. 2005, 55, 565-576.

113 C. Fonseca, S. Simoes, R. Gaspar, Paclitaxel-loaded PLGA nanoparticles: Preparation, physicochemical characterization and in vitro antitumoral activity, J. Control. Release 2002, 83, 273-286.

114 S.S. FenG, L. Mu, K.Y. Win, G. HUANG, Nanoparticles of biodegradable polymers for clinical administration of paclitaxel, Curr. Med. Chem. 2004, 11, 413-424.

115 Z. Lu, T.K. Yeh, M. Tsai, J.L. Au, M.G. Wientjes, Paclitaxel-loaded gelatin nanoparticles for intravesical bladder cancer therapy, Clin. Cancer Res. 2004, 10, 7677-7684.

116 T. Hamaguchi, Y. Matsumura, M. Suzuki, K. Shimizu, R. Goda, I. Nakamura, I. NaKatomi, M. Yokoyama, K. Кataoka, T. Kakizoe, NK105, a paclitaxel-incorporating micellar nanoparticle formulation, can extend in vivo antitumour activity and reduce the neurotoxicity of paclitaxel, Br. J. Cancer 2005, 92, 1240-1246.

117 S.K. SAHoo, W. MA, V. LabHaSeTwar, Efficacy of transferrin-conjugated paclitaxel-loaded nanoparticles in a murine model of prostate cancer, Int. J. Cancer 2004, 112, 335-340.

118 Z. Xu, W. Gu, J. Huang, H. Sui, Z. Zhou, Y. YANG, Z. Yan, Y. LI, In vitro and in vivo evaluation of actively targetable nanoparticles for paclitaxel delivery, Int. J. Pharm. 2005, 288, 361368

119 J.M. Koziara, P.R. Lockman, D.D. Allen, R.J. Mumper, Paclitaxel nanoparticles for the potential treatment of brain tumors, $J$. Control. Release 2004, 99, 259-269.

120 J. Zhou, F.Q. Zeng, C. Li, Q.S. Tong, X. GaO, S.S. XIE, L.Z. Yu, Preparation of arsenic trioxide-loaded albuminutes immuno-nanospheres and its specific killing effect on bladder cancer cell in vitro, Chin. Med. J. 2005, 118, 50-55.

121 C. Pellizzaro, D. Coradini, S. Morel, E. Ugazio, M.R. Gasco, M.G. DaIdone, Cholesteryl butyrate in solid lipid nanoparticles as an alternative approach for butyric acid delivery, Anticancer Res. 1999, 15, 3921-3926.

122 B. Salomone, R. Ponti, M.R. Gasco, E. Ugazio, P. Quaglino, S. OsellaAbate, M.G. Bernengo, In vitro effects of cholesteryl butyrate solid lipid nanoparticles as a butyric acid pro-drug on melanoma cells: Evaluation of antiproliferative activity and apoptosis induction, Clin. Exp. Metastasis 2001, 18, 663-673.

123 L. Serpe, S. Laurora, S. Pizzimenti, E. Ugazio, R. Ponti, R. Canaparo, F. Briatore, G. BARrera, M.R. 
Gasco, M.G. Bernengo, M. EAndi, G.P. ZARA, Cholesteryl butyrate solid lipid nanoparticles as a butyric acid pro-drug: Effects on cell proliferation, cell-cycle distribution and c-myc expression in human leukemic cells, Anticancer Drugs 2004, 15, 525-536.

124 M. Ceggnar, A. Premzl, V. ZavasnikBergant, J. Kristl, J. Kos, Poly(lactide-co-glycolide) nanoparticles as a carrier system for delivering cysteine protease inhibitor cystatin into tumor cells, Exp. Cell Res. 2004, 301, 223-231.

125 M. Michaelis, K. Langer, S. Arnold, H.W. Doerr, J. Kreuter, J. JR. Cinatl, Pharmacological activity of DTPA linked to protein-based drug carrier systems, Biochem. Biophys Res. Commun. 2004, 323, 1236-1240.

126 P. Beck, J. Kreuter, R. Reszka, I. Fichtn ER, Influence of polybutylcyanoacrylate nanoparticles and liposomes on the efficacy and toxicity of the anticancer drug mitoxantrone in murine tumour models, J. Microencapsul. 1993, 10, 101-114.

127 R. Reszka, P. Beck, I. Fichtner, M. Hentschel, L. Richter, J. Kreuter, Body distribution of free, liposomal and nanoparticle-associated mitoxantrone in B16-melanomabearing mice, J. Pharm. Exp. Ther. 1997, 280, 232-237.

128 R.L. Juliano, S. Alahari, H. Yoo, R. Kole, M. Сho, Antisense pharmacodynamics: Critical issues in the transport and delivery of antisense oligonucleotides, Pharm. Res. 1999, 16, 494-502.

129 G. Schwab, C. Chavany, I. Duroux, G. Goubin, J. Lebeau, C. Hélène, T. Saison-Behmoaras, Antisense oligonucleotides adsorbed to polyalkylcyanoacrylate nanoparticles specifically inhibit mutated Ha-rasmediated cell proliferation and tumorigenicity in nude mice, Proc. Natl. Acad. Sci. U.S.A. 1994, 91, 10460-10 464

130 G. Godard, A.S. Boutorine, E. SAISON-Behmoaras, C. HÉLÈn, Antisense effect of cholesterol- oligodeoxynucleotide conjugates associated with poly(alkylcyanoacrylate) nanoparticles, Eur. J. Biochem. 1995, 232, 404-410.

131 L. Tondelli, A. Ricca, M. Laus, M. Lelli, G. Citro, Highly efficient cellular uptake of c-myb antisense oligonucleotides through specifically designed polymeric nanospheres, Nucleic Acids Res. 1998, 26, 54255431.

132 G. Lambert, E. Fattal, H. PintoAlphandary, A. Gulik, P. Couvreur, Polyisobutylcyanoacrylate nanocapsules containing an aqueous core as a novel colloidal carrier for the delivery of oligonucleotides, Pharm. Res. 2000, 17, 707-714.

133 G. Lambert, J.R. Bertrand, E. Fattal, F. Subra, H. PintoAlphandary, C. Malvy, C. Auclair, P. Couvreur, EWS Fli-1 antisense nanocapsules inhibits Ewing sarcomarelated tumor in mice, Biochem. Biophys. Res. Commun. 2000, 279, 401-406.

134 G. Lambert, E. Fattal, P. Couvreur, Nanoparticulate systems for the delivery of antisense oligonucleotides, Adv. Drug Deliv. Rev. 2001, 47, 99-112.

135 B. Gopalan, I. Ito, C.D. Branch, C. Stephens, J.A. Roth, R. Ramesh, Nanoparticle based systemic gene therapy for lung cancer: Molecular mechanisms and strategies to suppress nanoparticle-mediated inflammatory response, Technol. Cancer Res. Treat. 2004, 3, 647-657.

136 L. Brannon-Peppas, J.O. Blanchette, Nanoparticle and targeted systems for cancer therapy, Adv. Drug Deliv. Rev. 2004, 56, 1649-1659.

137 M. Ogris, G. Walker, T. Blessing, R. Kircheis, M. Wolshek, E. WaGner, Tumor-targeted gene-therapy: Strategies for the preparation of ligand-polyethylene glycolpolyethyleneimine/DNA complexes, J. Control. Release 2003, 91, 173-181.

138 S.H. Pun, F. Tack, N.C. Belloce, J. Cheng, B.H. Grubbs, G.S. Jensen, M.E. Davis, M. BRewSTer, M. Janicot, B. Janssens, W. Floren, A. BAKKER, Targeted delivery of RNA- 
cleaving DNA enzyme (DNAzyme) to tumor tissue by transferrin-modified, cyclodextrin-based particles, Cancer Biol. Ther. 2004, 3, 641-650.

139 R.M. Schiffelers, A. Ansari, J. Xu, Q. Zhou, Q. TANG, G. Storm, G.
References $\mid 39$

Molema, P.Y. Lu, P.V. Scaria, M.C. Woodie, Cancer siRNA therapy by tumor selective delivery with ligandtargeted sterically stabilized nanoparticle, Nucleic Acids Res. 2004, 32, 149. 